\title{
Ambiguous Business Cycles: A Quantitative Assessment*
}

\author{
Sumru Altug ${ }^{\dagger, 1}$, Fabrice Collard ${ }^{2}$, Cem Çakmaklı, ${ }^{3}$, Sujoy Mukerji ${ }^{4}$, and Han Özsöylev ${ }^{3,5}$ \\ ${ }^{1}$ American University of Beirut and CEPR \\ ${ }^{2}$ University of Toulouse and CEPR \\ ${ }^{3}$ Koç University \\ ${ }^{4}$ Queen Mary University of London \\ ${ }^{5}$ University of Oxford
}

June 16,2020

\begin{abstract}
In this paper, we examine the cyclical dynamics of a Real Business Cycle model with ambiguity averse consumers and investment irreversibility using the smooth ambiguity model of Klibanoff et al. (2005, 2009). Ambiguity of belief about the productivity process arises as agents do not know the process driving variation in aggregate TFP, and they must make inferences regarding the true process at the same time as they infer the behavior of the unobserved temporary component using a Kalman filtering algorithm. Our findings may be summarized as follows. First, the standard business cycle facts hold in our framework, which are not altered significantly by changes in the degree of ambiguity aversion. Second, we demonstrate a role for information and learning effects, and show that lower initial ambiguity or greater confidence coupled with learning dynamics lowers the volatility and increases the persistence in all of the key macroeconomic variables. Third, comparing the performance of our model to the New Keynesian business cycle model of Ilut and Schneider (2014) with maxmin expected utility, we find that the version of their model without nominal and real frictions turns out to have limited success at matching the moments for the quantity variables. In the maxmin expected utility framework, the worst case scenario instills too much caution on the part of agents who, in the absence of a key set of nominal and real frictions, end up excessively reducing their responses to TFP shocks.
\end{abstract}

Keywords: Ambiguity, ambiguity aversion, information and learning, investment irreversibility, Real Business Cycles, New Keynesian model.

JEL Classification: C6, D8, E2

${ }^{*}$ We thank Nicholas Bloom, Benjamin Born, Steve Davis, Alan Paquet, Levent Koçkesen and two anonymous referees for helpful comments. An earlier version of this paper was presented at the Third Research Conference of the Macroeconomic Modelling and Model Comparison Network (MMCN), Frankfurt, 2019; the Asia-Pacific Economic Association (APEA) Conference, Los Angeles, 2018; Session on Prices, Business Fluctuations and Cycles at the International Atlantic Economic Society Meetings, Montreal, 2017; the $6^{\text {th }}$ Delhi Macroeconomics Workshop at the Indian Statistical Institute, Delhi, India, 2017; the Central Bank of the Republic of Turkey, 2017; and seminars at Groningen University, Koç University, Özyeğin University and American University Beirut. This research was supported by a Newton Mobility Grant from the British Academy, Grant Number NG160021. Fabrice Collard acknowledges funding from the French National Research Agency (ANR) under the Investments for the Future program (Investissements d'Avenir, grant ANR-17-EURE-0010).

${ }^{\dagger}$ Correspondence to: Sumru Altug, American University of Beirut, P.O.Box 11-0236 / Riad El-Solh / Beirut 1107 2020, Lebanon , e-mail: sa287@aub.edu.lb 


\section{Introduction}

In this paper we adopt the smooth ambiguity preferences of Klibanoff et al. $(2005,2009)$ to study the cyclical dynamics of a Real Business Cycle model with investment irreversibility and labor augmenting technology shocks. We examine the evolution of beliefs under ambiguity, information and learning and their impact on the cyclical behavior of key aggregate variables (such as output, consumption, investment and hours worked). In this framework, the shock to aggregate TFP evolves as a function of a latent variable governing its persistence. Ambiguity of belief about the productivity process arises in our framework as agents do not know whether variation in aggregate TFP is driven by a process with high persistence and low volatility, or one with lower persistence but higher volatility, and they must, at the same time, infer the behavior of the unobserved temporary component using a Kalman filtering algorithm. In our framework, time-varying uncertainty shocks that emerge under learning and ambiguity aversion interact with the irreversible nature of investment, leading to a wait-and-see attitude in response to uncertainty shocks and in turn, creating business cycle-like effects.

In the smooth ambiguity model it is possible to hold the agents' information fixed while varying their ambiguity attitude; the variation may range from extreme aversion (when it corresponds to maxmin expected utility) through a continuum of intermediate cases to ambiguity neutrality (i.e., expected utility with Bayesian model averaging). This facilitates a natural way to understand the pure effect of introducing ambiguity aversion into the environment. In essence, we are motivated by two questions. First, to what extent the results that were achieved in matching the dynamics of the equity premium, as studied by Collard et al. (2018), by moving from Bayesian model averaging to ambiguity aversion in an endowment economy is replicated in explaining the cyclical dynamics of real variables such as consumption, investment, output and hours. Second, in comparison to recent papers that study related questions, we seek to clarify the effect of changes in ambiguity and/or ambiguity aversion on dynamics of real variables in a neoclassical environment that is very minimally, if at all, perturbed by frictions, to better understand the effect of ambiguity in and of itself.

Our major results may be summarized as follows. First, the standard business cycle facts hold 
in our model, and they are not significantly altered by changes in the degree of ambiguity aversion. Following Tallarini (2000) and Backus et al. (2015), this result arises from the complete markets assumption that underlies the allocations of the social planning problem and the precautionary saving motives that it induces. Second, changes in initial ambiguity as measured by the degree of informativeness of the unknown latent process driving temporary fluctuations in productivity growth have significant business cycle effects. This demonstrates a role for information and learning in the smooth ambiguity model 11 Holding the degree of ambiguity aversion constant, lower initial ambiguity or greater confidence lead to significantly lower cyclical variability in investment and hours worked. Lower ambiguity also leads to the lower responsiveness of output and consumption to shocks as well as amplifying co-movement.

Third, we compare our findings with those from the New Keynesian model with maxmin expected utility studied by Ilut and Schneider (2014) $\mathrm{L}^{2}$ Based on a comparison of the model-generated moments from their model with those in the data, we find that removing the different types of frictions in their model has non-negligible effects on the results. In particular, a version of their model without nominal and real frictions, which corresponds more closely to our RBC framework, has limited success at matching the moments for the quantity variables. In the maxmin expected utility framework, which corresponds to the limiting case of the smooth ambiguity model as ambiguity aversion goes to infinity, agents make decisions based on the worst case distribution characterizing the TFP process. By contrast, in our framework, agents have endogenously distorted beliefs that depend on the properties of the unknown distributions characterizing TFP growth. In the absence of rigidities deriving from monopolistic competition in goods and labor supply and price and wage setting by firms of intermediate goods and by households, respectively, the worst case scenario instills too much caution on the part of agents, who end up excessively reducing their response to shocks from the estimated TFP process. These results indicate that the smooth ambiguity preferences together with the endogenous sources of pessimism that this framework generates are able to account for the cyclical dynamics of key quantity variables that the model with worst-case beliefs cannot capture.

\footnotetext{
${ }^{1}$ That learning dynamics may prove useful for generating the observed business cycle dynamics is noted by Backus et al. (2015).

${ }^{2}$ For recent experimental evidence on discriminating between the smooth ambiguity model and maxmin expected utility, see Cubitt et al. (2020).
} 
The recent literature on models with ambiguity and ambiguity aversion has examined a variety of phenomena. A group of papers has examined asset pricing relations under smooth ambiguity preferences. These include Ju and Miao (2007, 2012), who consider the implications of the generalized recursive smooth ambiguity model that distinguishes among risk aversion, intertemporal substitution and ambiguity aversion in an endowment economy or Jahan-Parvar and Liu (2014) and Liu and Zhang (2018), who extend this framework to a production economy. Collin-Dufresne et al. (2016) and Collard et al. (2018) exploit learning dynamics to generate significant effects of time-varying ambiguity on asset prices. Others have examined business cycle phenomena under alternative assumptions about the existence of real and nominal frictions; see, for example, Bidder and Smith (2012), Ilut and Schneider (2014) or Bhandari et al. (2016). Nimark (2014) presents a business cycle model with higher order beliefs and considers the impact of signals observed after unusual events that increase uncertainty and disagreement among agents. In all of these analyses, the presence of ambiguity and agents' aversion to ambiguity is taken as a key feature of the environment, as in our framework, and the focus of the analysis is to unravel the effects of these features on different outcomes.

The analysis of Jahan-Parvar and Liu (2014), Liu and Zhang (2018), and Bidder and Smith (2012) remains within a standard neoclassical environment describing investment and production. Bidder and Smith (2012) consider a Real Business Cycle Model with a version of the multiplier preferences that are robust to misspecification following Hansen and Sargent (2008) and find small but significant effects on business cycle moments of time-varying volatility shocks to TFP growth. In Ilut and Schneider (2014) or Bhandari et al. (2016), ambiguous preferences are paired with real and nominal frictions to account for the impact of changes in beliefs on cyclical outcomes $\mathrm{H}^{3}$ Our paper adds to this literature by examining the implications of a Real Business Cycle model with smooth ambiguity preferences and comparing them with the implications from a New Keynesian model with maxmin expected utility.

In the literature on ambiguity and/or robust decision-making, the issue of the extent of knowledge possessed by the econometrician versus agents a plays a key role; see Hansen (2007). In the New

\footnotetext{
${ }^{3}$ The concepts of ambiguity and ambiguity aversion, has also attracted considerable interest in recent years following the Great Recession of 2008-9. Some recent examples that examine the implications of ambiguity and ambiguity aversion for observed outcomes during the recent financial crisis include Zhou (2015), Dimmock et al. (2016) and Boyarchenko (2012).
} 
Keynesian models of Ilut and Schneider (2014); Bianchi et al. (2018), survey expectations are used to empirically discipline time variation in ambiguity. Other papers that use survey data to identify agents' beliefs include Bhandari et al. (2016) and Rossi et al. (2016). In our model, the agent and the econometrician possess the same information and must infer the nature of the true TFP growth process based on past observations of TFP growth. Thus, in our analysis, the rational expectations assumption that agents are endowed with more precise information than the econometrician is relaxed. This unifies the treatment of uncertainty and ambiguity without recourse to exogenous sources of data to estimate beliefs or extraneous assumptions about the knowledge possessed by agents versus the econometrician.

The remainder of this paper is organized as follows. Section 2 describes the nature of uncertainty and ambiguity and presents Bayesian inference of the underlying TFP process. Section 3 describes a Real Business Cycle model with ambiguity and ambiguity aversion while Section 4 presents the quantitative results obtained from this model. Section 5 compares our results with those from a New Keynesian with maxmin expected utility while Section 6 describes the results from other alternative frameworks. Section 7 concludes.

\section{The sources of uncertainty}

Uncertainty in this economy is assumed to be driven by the stochastic behavior of productivity growth. Specifically, there is a long-run average growth rate of productivity, $\bar{g}$, and a deviation from it, $x_{t+1}$, which is assumed to follow a persistent stochastic process. This specification of the technology process is similar to the models of long-run risk proposed by Bansal and Yaron (2004) and Croce (2014); see also Collard et al. (2018). However, the business cycle effect on productivity, $x_{t+1}$, is not observed directly and moreover, may derive from two possible processes. Specifically, the agent assumes that technology growth evolves as

$$
\begin{aligned}
g_{A, t+1} & =\bar{g}+x_{t+1}+\sigma_{A} \epsilon_{A, t+1}, \\
x_{t+1} & =\rho x_{t}+\sigma_{x} \epsilon_{x, t+1},
\end{aligned}
$$


where $\left(\epsilon_{A, t+1}, \epsilon_{x, t+1}\right)^{\prime} \sim N\left(0, I_{2}\right)$ and $I_{2}$ is a $2 \times 2$ identity matrix. Given these assumptions, next period's technology shock is written as

$$
A_{t+1}=A_{t} \exp \left(g_{A, t+1}\right)=A_{t} \exp \left(\bar{g}+x_{t+1}+\sigma_{A} \epsilon_{A, t+1}\right)
$$

According to this representation, the growth rate of the technology shock between $t$ and $t+1$ evolves as a function of the permanent mean, $\bar{g}$, the temporary component $x_{t+1}$, and some noise. At time $t$, the agent has available observations on the current and past values of the technology shock, $A_{t}$. However, the agent does not know the process generating $x_{t}$ and forms beliefs about it, given prior beliefs at time 0 and the observations on the technology as $A_{s}$ for $s=1, \ldots, t 4^{4}$

This process is parameterized using observations of aggregate TFP growth for the postwar period. Figure 1 displays the growth rates of the factor utilization adjusted TFP series measured (at annualized rates) for the full sample together with NBER recession dates in shaded areas $5^{5}$

Figure 1: The growth rate of factor utilization adjusted TFP (in percentage terms) over the sample 1947:2-2015:4

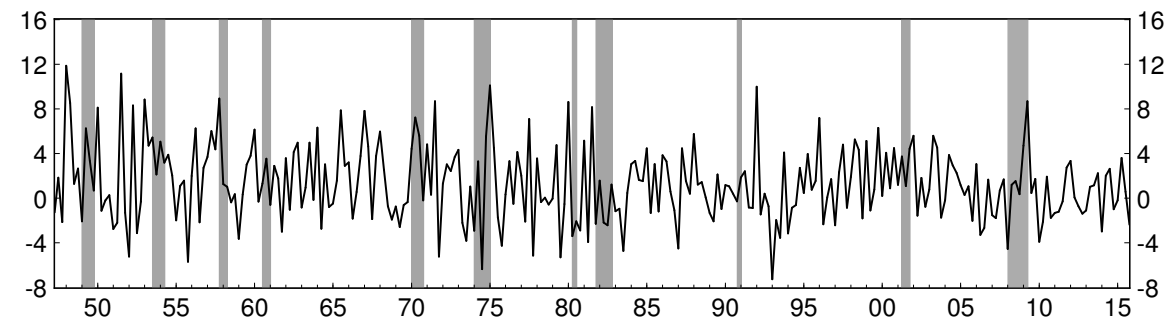

This figure shows that there have been significant variations in the average growth rate of productivity and its volatility over the sample period. As Gordon $(2015)$ and others have noted, there has been a secular decline in TFP growth in that average TFP growth has declined in the

\footnotetext{
${ }^{4}$ The uncertainty we assume derives from the nature of latent process driving productivity growth. However, similar formulations may be used to model uncertainty about other types of shocks such as investment-specific shocks; see, for example, Greenwood et al. (1997) or Fisher (2006).

${ }^{5}$ For the observations on (the growth rate of) the technology shock, $A_{t}$, we use seasonally adjusted data on total factor productivity (TFP) growth obtained from the Federal Reserve of San Francisco, see Fernald (2012) for details. The data on inputs, including capital, are used to produce a real-time, quarterly series on total factor productivity growth as the measured Solow residual. The advantages of these data are that they are adjusted to account for the changes in factor utilization and they are at the quarterly frequency, unlike the typical annual TFP data.
} 
post-1980's relative to the pre-1980's period from $1.78 \%$ to $0.88 \%$ for the adjusted TFP growth series, measured at annualized rates. There is also a decline in the variability of TFP growth after 1980 but this is not as large as the decline in the average quantities ${ }^{6}$

Consider the inference process of an agent who is a Bayesian learner regarding the properties of the unknown TFP growth and who faces these data. To exploit the business cycle effects in the TFP growth process, the agent is assumed to be situated in 1977, which implies that the agent has roughly 30 years of data to infer the parameters of the model in equations $(2.1+2.2) .7$ This corresponds to the part of the data set prior to the occurrence of the potential (ex-post) structural change in the observed TFP process. As the agent is a Bayesian learner regarding the distribution of the underlying parameters, s/he infers the values of the persistence/volatility pairs using a Bayesian rule by combining prior beliefs with the data, i.e. prior distributions of the parameters together with the likelihood function to form posterior distributions. The agent's ignorance about the model parameters is modeled using non-informative prior distributions about the model parameters. The estimated posterior distributions of the model parameters are displayed in Figure 2.

Figure 2: Parameter distributions estimated using the TFP growth process
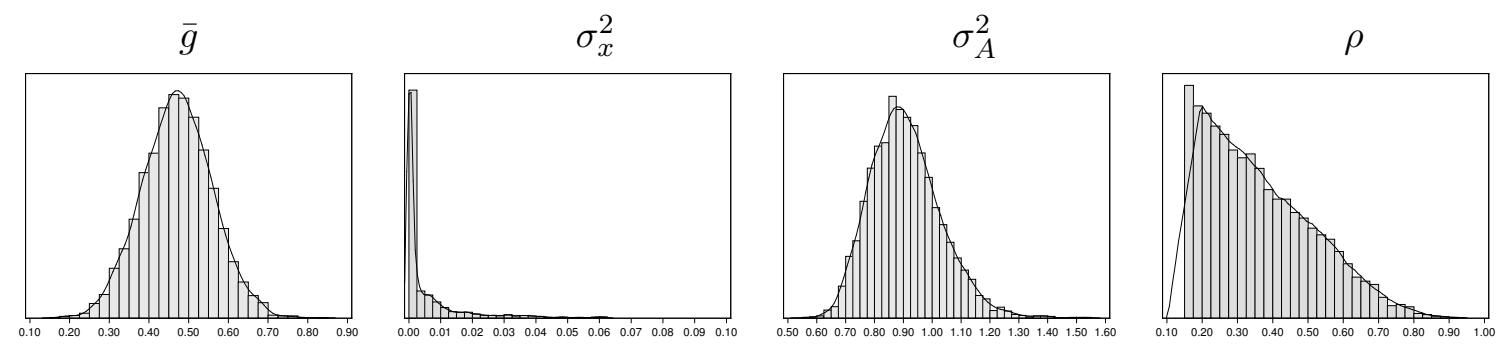

Figure 2 shows that the distribution of the model parameters are precisely estimated except for the persistence parameter, suggesting that the agent can infer the parameters $\bar{g}, \sigma_{x}^{2}$ and $\sigma_{A}^{2}$ by using the posterior distributions. However, the ambiguity confronting by the agent stems from the

\footnotetext{
${ }^{6}$ Specifically, the standard deviation of adjusted TFP growth has fallen from $3.75 \%$ in the pre-1980's period to to $2.94 \%$ in the post-1980's period. Similar findings hold for the unadjusted TFP growth series, which are not reported for the sake of brevity.

${ }^{7}$ See Collard et al. (2018) for a similar setup.
} 
persistence parameter (and therefore, from $x_{t}$ ) as the persistence parameter $\rho$ covers a wide range of values between 0 and and 0.90 with high probability. This also indicates the difficulty to pinpoint the exact value of the persistence in the TFP growth process. To see this further, next, we estimate the models for given values of $\rho$ covering this range, i.e. $\rho=0.25,0.30,0.65,0.70,0.85,0.90$. The results are displayed in Table 1.

Table 1: Posterior results for the model using TFP-util for different values of $\rho$ using the sample of 1947-2 : 1977-4

\begin{tabular}{lcccccc}
\hline \hline & & & & & & \\
$\rho$ & $\underline{0.25}$ & $\underline{0.30}$ & $\underline{0.65}$ & $\underline{0.70}$ & $\underline{0.85}$ & $\underline{0.90}$ \\
$\bar{g}$ & $0.469(0.086)$ & $0.469(0.086)$ & $0.469(0.086)$ & $0.469(0.086)$ & $0.469(0.086)$ & $0.469(0.086)$ \\
$\sigma_{A}$ & $0.945(0.075)$ & $0.946(0.071)$ & $0.949(0.063)$ & $0.950(0.063)$ & $0.952(0.062)$ & $0.953(0.062)$ \\
$\sigma_{x}$ & $0.046(0.120)$ & $0.044(0.110)$ & $0.056(0.080)$ & $0.054(0.073)$ & $0.040(0.049)$ & $0.033(0.040)$ \\
\hline Max. Like. & -167.40 & -167.40 & -167.40 & -167.40 & -167.40 & -167.40 \\
Mar. Like. & -169.70 & -169.73 & -170.16 & -170.20 & -170.32 & -170.38 \\
\hline
\end{tabular}

Note: The results show the posterior means and posterior standard deviations (in parenthesis) of the model parameters in 2.1)-2.2 (evaluated in percentage terms). The inference was carried out with 60,000 draws where the first 10,000 are used as burn-in sample. We kept every $5^{\text {th }}$ draw, which yields a sample of 10,000 draws from the ergodic distribution. Max. Like. refers to the maximized likelihood value of the models, whereas Mar. Like refers to the marginal likelihood value of the models, i.e. independent of the specific parameter values except the value of the $\rho$.

Table 1 provides important insights regarding the ambiguity the agent is facing. While the estimated value of the $\bar{g}$ remains unchanged, there are some rather minor changes in the estimates of the variance parameters. Regardless of the values of the $\rho$ ranging from 0.20 to 0.90 , the values of (logarithm of the) maximum likelihood, which is computed using the posterior mode of the distributions, do not change at all. Hence, different processes depending on the value of persistence parameter yield the same likelihood 8 This implies that the agent (and, by analogy, the econometrician) faces ambiguity about the process stemming from the fact that the value of the persistence parameter cannot be inferred precisely.

\footnotetext{
${ }^{8}$ We also compute the (logarithm of the) marginal likelihood values of the models. Note that the marginal likelihood is computed by integrating out the parameter distributions taking parameter uncertainty into account. Therefore, it provides a precise metrics of model probabilities as it is independent of the parameter values except the value of persistence parameter, $\rho$. The changes in the marginal likelihood values are very minor, only to the scale of 0.60 in terms of the log-differences.
} 


\section{$2.1 \quad$ Beliefs}

The evidence in the previous section suggests a natural way to model the ambiguity that agents are facing. This involves assuming that agents are unsure about the value of the persistence parameter that determines the evolution of the latent productivity process, which they believe could be high $\left(\rho_{h}\right)$ or low $\left(\rho_{l}\right)$. For a given $\left(x_{k, t}, \rho_{k}\right)$ for $k=l, h$ and the current observations, the probability distribution over $g_{A_{k}, t+1}$ is given by

$$
g_{A_{k}, t+1} \sim N\left(\bar{g}+\rho_{k} x_{k, t}, \sigma_{A_{k}}^{2}+\sigma_{x_{k}}^{2}\right) .
$$

which is the first-order uncertainty the agent confronts as discussed earlier.

Denote by $\eta_{t}$ as the agent's belief about the true DGP denoted for the low persistence process with $\rho=0.30$ relative to the one with $\rho=0.85$. This is computed using the agent's updating mechanism after observing data on the actual TFP growth process 99 The sequence of $\eta_{t}$ 's is computed over the period starting from 1947:12 until 2015:4 using the parameter setup as shown in Table 1.

Figure 3: Evolution of the probability of the model with $\rho=0.30$ being the true DGP over the sample 1947:2:2015-4

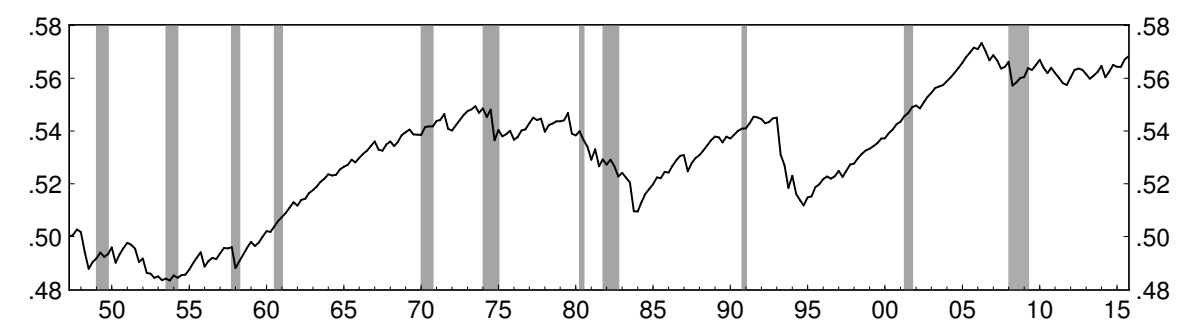

Figure 3 displays the evolution of these beliefs. In line with the results so far, the probabilities attached to each separate process vary in a band around 0.50-0.55, with some tendency to increase above this value towards the end of the sample. This suggests that there is very little learning that is occurring over the sample period, though there is an increase in the probability attached to the low persistence process in the run-up to the 2008 global financial crisis.

\footnotetext{
${ }^{9}$ The steady state Kalman filter is used to compute the beliefs, the details of which are provided in Section B of the Supplementary Material.
} 
Figure 4: Filtered values of the deviations, $x_{t}$, from the long-run over the sample 1947:2:2015-4

Filtered values of $x_{t}\left(* 10^{-3}\right)$ for the DGP with $\rho=0.30, \hat{x}_{l, t}$

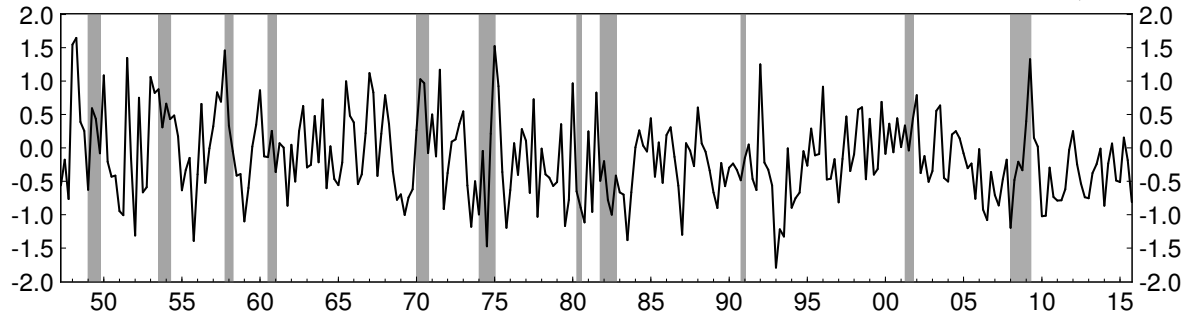

Filtered values of $x_{t}$ for the DGP $\rho=0.85, \hat{x}_{h, t}$

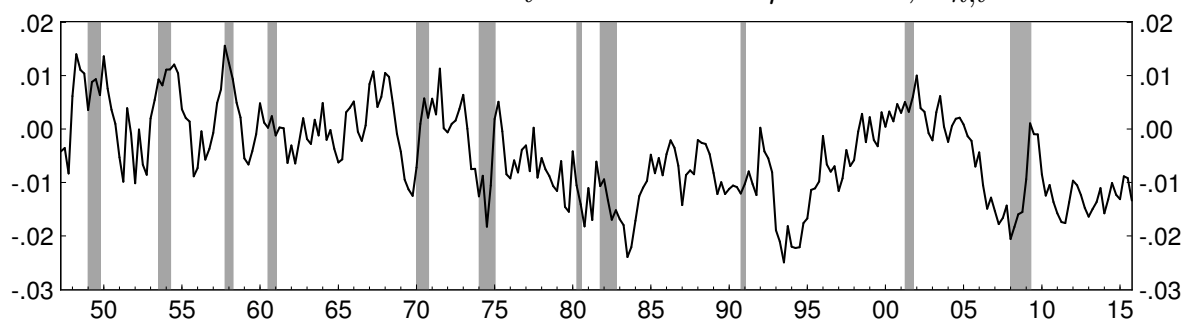

The two panels in Figure 4 further show the time series of the filtered means, $\hat{x}_{l, t}$ and $\hat{x}_{h, t}$ (in percentage terms) estimated using data on actual TFP growth. The filtered means tend to decline during the recessions of the 1970's and 1980's as well as during the global financial crisis of 2008. This decline is particularly severe for agents' beliefs regarding the cyclical mean of the high persistence process, $\hat{x}_{h, t}$. This is a priori evidence for the existence of a role for ambiguity in agents' decision problem. The reason is that an ambiguity-averse agent endogenously behaves as if the uncertainty is more persistent and severe following negative shocks than in normal times.

These results motivate the notion of second-order uncertainty confronted by the agent, which arises from the fact that agent cannot distinguish which process $x_{k, t}$ is drawn from. The support of the second-order distribution is a union of two component sets, $\left\{\rho_{l} x_{l, t} \mid x_{l, t} \in \Re\right\}$ and $\left\{\rho_{h} x_{h, t} \mid x_{h, t} \in \Re\right\}$. The agent's prior belief ascribes a measure to each component sets ${ }^{10}$ The agent updates her beliefs according to Bayes rule, based on the history of growth realizations and under the assumption that the economy conforms to one of the two processes described above. Let $\hat{x}_{k, t} \equiv E\left[x_{t} \mid g_{A_{k}, 1}, \ldots, g_{A_{k}, t}\right]$ denote the expectation of $x_{k, t}$, conditional on the history of growth rates up to $t$, i.e. filtered values of $x_{t}$, if the beliefs were updated assuming $\rho=\rho_{k}$ is the data generating process. The agent's posterior

\footnotetext{
${ }^{10}$ The measure on the first component is given by $\eta_{0} \times N\left(0, \sigma_{0}^{2}\right)$ and that on the second component by $\left(1-\eta_{0}\right) \times N\left(0, \sigma_{0}^{2}\right)$.
} 
beliefs are described by a measure on the first component set given by $\eta_{t} \times N\left(\hat{x}_{l, t}, \Omega_{l}\right)$ and that on the second by $\left(1-\eta_{t}\right) \times N\left(\hat{x}_{h, t}, \Omega_{h}\right)$, where $\Omega_{k}, k=l, h$ denotes the steady state variance associated with the Kalman filter based on the process with $\rho=\rho_{k}$. Hence, the agent's posterior beliefs may be summarized by the tuple $\mu_{t}=\left(\hat{x}_{l, t}, \hat{x}_{h, t}, \eta_{t}\right)$.

To summarize, ambiguity is the agent's uncertainty about the probability distribution governing the productivity process. While she knows the form of the distribution characterizing productivity, she does not know values of the persistence parameter, $\rho$, and the latent component of productivity, $x_{t}$, at any point in time and therefore, does not know the mean of the distribution generating productivity growth next period. In the framework of the smooth ambiguity model, this ambiguity is expressed as a (second-order) probability distribution over the values of $\left(\rho, x_{t}\right)$. The prior (or second-order probability) belief is the initial ambiguity. The ambiguity evolves with observations on productivity growth, as the agent learns, by adapting the prior belief to the observations. Hence, evolution of ambiguity is formally expressed by the sequence of posteriors. By exogenously changing how uncertain (alternatively, how informative or how confident) the prior belief is, the comparative statics of changes in the initial ambiguity ascribed to the agent may be traced out.

In this framework, the evolution of ambiguity is endogenous, it is only the specification of the initial ambiguity, the prior, that is exogenous. As ambiguity evolves, endogenously, it may increase or

decrease. This occurs because the posterior $\mu_{t}$ is a mixture distribution (a convolution) with weights $\left(\eta_{t}, 1-\eta_{t}\right)$. The two components of the mixture are Gaussian distributions with different volatilities. Hence, as the weights $\eta_{t}$ change because of updating, the volatility of the mixture changes, i.e., posterior ambiguity changes.

\section{A Real Business Cycle Model with ambiguity aversion}

We now embed this process of productivity growth and beliefs into a Real Business Cycle model with ambiguity aversion and investment irreversibility to understand its quantitative implications. Section 3.1 describes preferences and production, Section 3.2 describes the social planner's associated with the model while Section 3.3 describes its implications. 


\subsection{Preferences and the production technology}

The economy is a standard one-sector economy where the production function of the representative firm is given by

$$
y_{t}=k_{t}^{a}\left(A_{t} n_{t}\right)^{1-a}, \quad 0<a<1,
$$

where $A_{t}$ is the labor-augmenting technology shock as described in $(2.3), k_{t}$ is the beginning-of-period capital stock and $n_{t}$ is total hours. The firm's capital stock evolves as

$$
k_{t+1}=(1-\delta) k_{t}+i_{t},
$$

where $i_{t}$ is gross investment and $0<\delta<1$ is the depreciation rate. To introduce real frictions into the investment process, investment is assumed to be irreversible, $i_{t} \geq 0$. In contrast to the standard adjustment costs model, the presence of investment irreversibility leads to an endogenous cost of adjustment that varies with uncertainty and information possessed by the firm; for a further discussion, see Demers et al. (2003).

Agents' preferences are modeled using the dynamic, recursive version of the smooth ambiguity averse preferences developed by Klibanoff et al. (2005, 2009), denoted as KMM hereafter. This model is based on the state space, which is the set of all observation paths emanating from an initial state $s_{0}$. Thus, the state at date $t$ is denoted $s^{t}=\left(s_{0}, s_{1}, \cdots, s_{t}\right)$, where $s_{t} \in \Upsilon_{t}$. Agents choose consumption/investment plans $f$, each of which associates a payoff to the node $s^{t}$ in the event tree. The agent is uncertain about the stochastic process governing the probabilities on the event tree. This uncertainty is indexed by the parameter $\theta \in \Theta$, which denotes the set of unobservable parameters. The probability that the next observation will be $s_{t+1}$, given that the node $s^{t}$ has been reached on the event tree, is given by $\pi_{\theta}\left(s_{t+1} \mid s^{t}\right)$. The agent further has a prior $\mu(\theta)$ for $\theta \in \Theta$.

Using the representation in KMM, the recursive smooth ambiguity preferences over plans $f$ at 
the node $s^{t}$ are updated and represented as

$$
V_{s^{t}}(f)=u\left(f\left(s^{t}\right)\right)+\beta \phi^{-1}\left[\int_{\Theta} \phi\left(\int_{\Upsilon_{t+1}} V_{\left(s^{t}, s_{t+1}\right)}(f) d \pi_{\theta}\left(s_{t+1} \mid s^{t}\right)\right) d \mu\left(\theta \mid s^{t}\right)\right],
$$

where $V_{s^{t}}(f)$ is a recursively defined direct value function, $u(\cdot)$ characterizes attitudes towards risk, $\beta$ is a discount factor, $\phi(\cdot)$ is a function characterizing the agent's ambiguity attitude, and $\mu\left(\cdot \mid s^{t}\right)$ denotes the Bayesian posterior. The model does not, in general, allow for a reduction between the second-order beliefs $\mu$ and the first-order probabilities denotes by $\pi_{\theta}$ in terms of the predictive distribution for $s_{t+1}$, given $\theta$; such a reduction occurs only in the case of a linear $\phi$ which represents an ambiguity neutral Bayesian expected utility maximizer. Klibanoff et al. (2005) show that ambiguity aversion is defined as an aversion to mean preserving spreads in the distribution of expected utilities induced by agent's prior beliefs under a specific action, which corresponds to $-\phi^{\prime \prime}(x) x / \phi^{\prime}(x)$; hence, a concave function, $\phi(\cdot)$, characterizes ambiguity aversion. Risk aversion, as usual, is inferred from the properties of the function, $u(\cdot)$.

An alternative specification of ambiguous preferences is provided by the maxmin expected utility framework of Gilboa and Schmeidler (1989). According to this specification, agents act as if they evaluate plans using a worst case probability drawn from a set of multiple beliefs. In the static case, Klibanoff et al. (2005) show that the maxmin expected utility model, $\min _{\pi \in \Pi} E_{\Pi} u(C)$, is a limiting case of the smooth ambiguity model, $E_{\mu} \phi\left(E_{\pi} u(C)\right)$, when ambiguity aversion goes to infinity. An important advantage of the smooth ambiguity model over other models of ambiguity is that comparative statics regarding ambiguity attitudes can be analyzed using the $\phi$ function, holding ambiguity fixed. This is in contrast to the multiple priors framework where the set of priors $\Pi$ in that model characterizes ambiguity and ambiguity attitudes.

One issue with the smooth ambiguity model is that it cannot separately identify attitudes towards risk aversion, intertemporal substitution, and ambiguity aversion. Following Kreps and Porteus (1978) and Epstein and Zin (1989), Ju and Miao (2012) propose the generalized recursive ambiguity utility model that allows for a three-way separation among risk aversion, ambiguity aversion, and 
intertemporal substitution.11 In Section 4, we discuss the role of risk aversion, intertemporal substitution and ambiguity aversion for cyclical quantities and prices while in Section 5, we compare the implications of the smooth ambiguity model relative to the model with maxmin expected utility for business cycle fluctuations.

\subsection{The social planner's problem}

Given the stochastic growth in the technology shock, the state variables for the social planner's problem consist of the initial capital $k_{t}$, initial beliefs $\mu_{t}$ and the level of the technology shock, $A_{t}$. Define the value function for the generic social planner's problem by $J\left(k_{t}, \mu_{t}, A_{t}\right)$.

In what follows, we assume that the current-period utility function, $u$, and the ambiguity aversion function, $\phi$, are described by the power-power specification as $u(c, l)=\left(c^{\nu} l^{1-\nu}\right)^{1-\gamma} /(1-\gamma), \gamma \geq 0$, $0 \leq \nu \leq 1$ and $\phi(x)=x^{1-\alpha} /(1-\alpha), \alpha \geq 012$ Define $\left\{\hat{c}_{t}, \hat{i}_{t}, \hat{k}_{t}, \hat{y}_{t}\right\}=\left\{c_{t} / A_{t}, i_{t} / A_{t}, k_{t} / A_{t}, y_{t} / A_{t}\right\}$. Under stationarity-inducing transformation, the value function for the social planner's problem may be expressed as $J\left(k_{t}, \mu_{t}, A_{t}\right)=\hat{J}\left(\hat{k}, \mu_{t}\right) A_{t}^{(1-\gamma) \nu}$.

Using this relation, the indirect value function for the transformed problem is given by

$$
\hat{J}\left(\hat{k}_{t}, \mu_{t}\right)=\max _{\hat{c}_{t}, n_{t}, \hat{i}_{t}}\left\{\frac{\left(\hat{c}_{t}^{\nu} l_{t}^{1-\nu}\right)^{1-\gamma}}{1-\gamma}+\beta\left[E_{\mu_{t}}\left(E_{x_{t}} \hat{J}\left(\hat{k}_{t+1}, \mu_{t+1}\right) \exp \left(\nu(1-\gamma) g_{A, t+1}\right)\right)^{1-\alpha}\right]^{\frac{1}{1-\alpha}}\right\}
$$

subject to $\hat{c}_{t}+\hat{i}_{t} \leq \hat{k}_{t}^{a} n_{t}^{1-a}, \exp \left(g_{A, t+1}\right) \hat{k}_{t+1}=(1-\delta) \hat{k}_{t}+\hat{i}_{t}, l_{t}+n_{t} \leq 1, \hat{i}_{t} \geq 0$ and the law of motion for beliefs. In this specification, we restrict $0<\gamma<1$ and $\gamma<\alpha$, which ensures ambiguity aversion 13

The social planner's problem may be written in an alternative by noting that the initial beliefs of the agent are given by the tuple $\mu_{t}=\left(\hat{x}_{l, t}, \hat{x}_{h, t}, \eta_{t}\right)^{\prime}$. To describe the evolution of future beliefs,

\footnotetext{
${ }^{11}$ Strzalecki (2013) shows that there is interdependence between ambiguity and the timing of the resolution of uncertainty in models of ambiguity aversion, and that a quantitative assessment is required to disentangle the importance of two effects, which may depend on the calibrated parameters in applications such as ours.

${ }^{12}$ This facilitates the implementation of a stationarity inducing transformation, which exists in the power-power and log-exponential cases. See Ju and Miao (2007) and Section A of the Supplementary Material.

${ }^{13}$ It is possible to replicate the solution to the social planner's problem in a recursive competitive equilibrium where the representative consumer makes consumption and labor supply choices and holds shares and bonds in the firm while all production and capital accumulation decisions are made by value-maximizing firms; see Altug and Labadie (2008), Ch. 10 for a discussion. Also see Kaltenbrunner and Lochstoer (2010) for a similar decentralization scheme in the context of an economy with non-expected utility preferences.
} 
denote by $\hat{x}_{k, t+1}^{(i)}, i=l, h$ and $k=l, h$, the agent's forecast for the one-period ahead update using a Kalman filter which takes the model with $\rho=\rho_{k}$ as the data generating process, when the data is actually generated by the $\rho=\rho_{i}$ process. Correspondingly, $\eta_{t+1}^{(l)}\left(\right.$ respectively, $\left.\eta_{t+1}^{(h)}\right)$ is the posterior probability that the low persistence process is the correct model when the low (high) persistence process is the data generating process. Using this representation of beliefs, the indirect value function may be expressed as

$$
\begin{aligned}
\hat{J}\left(\hat{k}_{t}, \hat{x}_{l, t}, \hat{x}_{h, t}, \eta_{t}\right)=\max _{\hat{c}_{t}, n_{t}, \hat{i}_{t}}\left\{\frac{\left(\hat{c}_{t}^{\nu} l_{t}^{1-\nu}\right)^{1-\gamma}}{1-\gamma}+\right. \\
\beta\left[\eta_{t} E_{\hat{x}_{l, t}}\left(E_{x_{l, t}} \hat{J}\left(\hat{k}_{t+1}^{(l)}, \hat{x}_{h, t+1}^{(l)}, \hat{x}_{l, t+1}^{(l)}, \eta_{t+1}^{(l)}\right) \exp \left(\nu(1-\gamma) g_{A_{l}, t+1}\right)\right)^{1-\alpha}+\right. \\
\left.\left.\quad\left(1-\eta_{t}\right) E_{\hat{x}_{h, t}}\left(E_{x_{h, t}} \hat{J}\left(\hat{k}_{t+1}^{(h)}, \hat{x}_{h, t+1}^{(h)}, \hat{x}_{l, t+1}^{(h)}, \eta_{t+1}^{(h)}\right) \exp \left(\nu(1-\gamma) g_{A_{h}, t+1}\right)\right)^{1-\alpha}\right]^{\frac{1}{1-\alpha}}\right\}
\end{aligned}
$$

subject to $\hat{c}_{t}+\hat{i}_{t} \leq \hat{k}_{t}^{a} n_{t}^{1-a}, \exp \left(g_{A, t+1}\right) \hat{k}_{t+1}=(1-\delta) \hat{k}_{t}+\hat{i}_{t}, l_{t}+n_{t} \leq 1$, and $\hat{i}_{t} \geq 0$ and given the laws of motion for beliefs $x_{k, t+1}^{(i)}$ and the condition determining the evolution of $\eta_{t+1}^{(i)}$ for $i=l, h{ }^{14}$

\subsection{The role of endogenous pessimism}

To further understand the distortions inherent in the model with ambiguity and ambiguity aversion, define the quantities $\Lambda_{t}$ and $\xi_{i, t}$ as ${ }^{15}$

$$
\begin{aligned}
\Lambda_{t}= & {\left[\sum_{i=l, h} \eta_{i, t} E_{\hat{x}_{i, t}}\left(E_{x_{i, t}} \hat{J}\left(\hat{k}_{t+1}^{(i)}, \hat{x}_{h, t+1}^{(i)}, \hat{x}_{l, t+1}^{(i)}, \eta_{t+1}^{(i)}\right) \exp \left(\nu(1-\gamma) g_{A_{i}, t+1}\right)\right)^{-\alpha}\right] \div } \\
& {\left[\sum_{i=l, h} \eta_{i, t} E_{\hat{x}_{i, t}}\left(E_{x_{i, t}} \hat{J}\left(\left(\hat{k}_{t+1}^{(i)}, \hat{x}_{h, t+1}^{(i)}, \hat{x}_{l, t+1}^{(i)}, \eta_{t+1}^{(i)}\right) \exp \left(\nu(1-\gamma) g_{A_{i}, t+1}\right)\right)^{1-\alpha}\right]^{\frac{-\alpha}{1-\alpha}}\right.}
\end{aligned}
$$

\footnotetext{
${ }^{14}$ See Section B of the Supplementary Material.

${ }^{15}$ To conserve on notation, let $\eta_{i, t}, i=l, h$ denote probability of the low (high) persistence process at time $t$ so that $\eta_{l, t}=\eta_{t}$ and $\eta_{h, t}=1-\eta_{t}$.
} 
and

$$
\begin{aligned}
\xi_{i, t}= & {\left[\left(E_{x_{i, t}} \hat{J}\left(\hat{k}_{t+1}^{(i)}, \hat{x}_{h, t+1}^{(i)}, \hat{x}_{l, t+1}^{(i)}, \eta_{t+1}^{(i)}\right) \exp \left(\nu(1-\gamma) g_{A_{i}, t+1}\right)\right)^{-\alpha}\right] \div } \\
& {\left[\sum_{i=l, h} \eta_{i, t} E_{\hat{x}_{i, t}}\left(E_{x_{i, t}} \hat{J}\left(\left(\hat{k}_{t+1}^{(i)}, \hat{x}_{h, t+1}^{(i)}, \hat{x}_{l, t+1}^{(i)}, \eta_{t+1}^{(i)}\right) \exp \left(\nu(1-\gamma) g_{A_{i}, t+1}\right)\right)^{-\alpha}\right] .\right.}
\end{aligned}
$$

Let $\lambda_{t}$ denote the Lagrange multiplier on the aggregate resource constraint and $\varphi_{t}$ the multiplier on the irreversibility constraint. Using the expressions for $\Lambda_{t}$ and $\xi_{k, t}$, the first-order conditions can be combined to yield the optimality conditions that characterize the choice of $c_{t}, l_{t}$ and $i_{t}$ as

$$
\begin{gathered}
\frac{1-\nu}{\nu} \frac{\hat{c}_{t}}{l_{t}}=(1-a) \hat{k}_{t}^{a} n_{t}^{-a} \\
\lambda_{t}-\varphi_{t}=\Lambda_{t} \sum_{i=l, h} \eta_{i, t} E_{\hat{x}_{i t}}\left[\xi_{i, t} E_{x_{i t}}\left(\hat{J}_{1}\left(\hat{k}_{t+1}^{(i)}, \hat{x}_{h, t+1}^{(i)}, \hat{x}_{l, t+1}^{(i)}, \eta_{t+1}^{(i)}\right) \exp \left((\nu(1-\gamma)-1) g_{A, t+1}\right)\right)\right] .
\end{gathered}
$$

Finally, the envelope condition is given by

$$
\hat{J}_{1}\left(\hat{k}_{t}, \hat{x}_{l, t}, \hat{x}_{h, t}, \eta_{t}\right)=\lambda_{t} a \hat{k}_{t}^{a-1} n_{t}^{1-a}+(1-\delta)\left(\lambda_{t}-\varphi_{t}\right)
$$

The conditions in (3.7) and (3.8) require that at the optimum, the marginal rate of substitution between consumption and leisure equals the real wage and that optimal investment is determined by equating the cost of investment today to its expected marginal benefit tomorrow, after accounting for the irreversibility constraint. Here $\lambda_{t}$ denotes the marginal utility of consumption at the optimum. Given a solution for $\hat{c}_{t}$ as a function of the current state variables $\left(\hat{k}_{t}, \hat{x}_{h t}, \hat{x}_{l t}, \eta_{t}\right)$, the condition in (3.7) can be solved for current $n_{t}$ for the current state variables as well. ${ }^{16}$

To further understand the determinants of optimal investment, define $\Lambda_{t+1}^{0}\left(\Lambda_{t+1}^{1}\right)$ and $\xi_{i, t+1}^{j}$ $\left(\xi_{i, t+1}^{1}\right)$ by the relevant expressions evaluated at the values of $\hat{i}_{t+1}=0\left(\hat{i}_{t+1}>0\right)$. Using the first-order conditions and the envelope condition that holds at the consumer's optimum, the envelope condition

\footnotetext{
${ }^{16}$ This solution is used in the numerical solution procedure together with the expression for the indirect value function in (3.4) to obtain the decision rules for $i_{t}$ and $n_{t}$.
} 
that holds at time $t+1$ may be written as

$$
\begin{aligned}
& \hat{J}_{1}\left(\hat{k}_{t+1}, \hat{x}_{h, t+1}, \hat{x}_{l, t+1}, \eta_{t+1}\right)=\lambda_{t+1} a \hat{k}_{t+1}^{a-1} n_{t+1}^{1-a}+(1-\delta) \lambda_{t+1} \min (1 \\
& \left.\Lambda_{t+1}^{0} \sum_{i=l, h} \eta_{i, t+1} E_{\hat{x}_{i, t+1}}\left[\xi_{i, t+1}^{0} E_{x_{i, t+1}}\left(\frac{\hat{J}_{1}\left((1-\delta) \hat{k}_{t+1}^{(i)}, \hat{x}_{h, t+2}^{(i)}, \hat{x}_{l, t+2}^{(i)}, \eta_{t+2}^{(i)}\right) \exp \left((\nu(1-\gamma)-1) g_{A_{i}, t+2}\right)}{\lambda_{t+1}}\right)\right]\right)
\end{aligned}
$$

The envelope condition provides an expression for the marginal value of capital next period, which affects the current investment decision today. In the presence of an irreversibility constraint, the marginal value of capital depends on whether the irreversibility constraint is binding or not next period. This occurs when $\lambda_{t+1}$ is greater than the expected value of the marginal value of capital next period, where the expectation reflects the existence of the distortions implied by the presence of ambiguity and ambiguity aversion. Thus, the existence of the irreversibility constraint leads to an endogenous risk premium or an option value to wait and creates a non-linear mechanism for the transmission of technology shocks in a simple Real Business Cycle framework.

Now consider the conditions describing the optimal choice of investment at time $t$.

$$
\begin{gathered}
\lambda_{t}=\Lambda_{t}^{1} \sum_{i=l, h} \eta_{i, t} E_{\hat{x}_{i t}}\left[\xi_{i, t}^{1} E_{x_{i, t}}\left(\hat{J}_{1}\left(\hat{k}_{t+1}^{(i)}, \hat{x}_{h, t+1}^{(i)}, \hat{x}_{l, t+1}^{(i)}, \eta_{t+1}^{(i)}\right) \exp \left((\nu(1-\gamma)-1) g_{A_{i}, t+1}\right)\right)\right] \\
\text { if } \hat{i}_{t}>0, \\
\lambda_{t}>\Lambda_{t}^{0} \sum_{i=l, h} \eta_{i, t} E_{\hat{x}_{i t}}\left[\xi_{i, t}^{0} E_{x_{i, t}}\left(\hat{J}_{1}\left((1-\delta) \hat{k}_{t}, \hat{x}_{h, t+1}^{(i)}, \hat{x}_{l, t+1}^{(i)}, \eta_{t+1}^{(i)}\right) \exp \left((\nu(1-\gamma)-1) g_{A_{i}, t+1}\right)\right)\right] \\
\text { if } \hat{i}_{t}=0,
\end{gathered}
$$

In our framework, ambiguity arises from the nature of the processes that generate TFP growth which, combined with the ambiguity averse preferences, leads to an endogenous tilting or distortion of the posterior distributions that signify agent's subjective beliefs about the validity of a given process describing the external environment.

To understand the role of ambiguity and ambiguity aversion in shaping these functions, consider two special cases:

- $\eta_{t} \rightarrow 1$. In this case, the agent knows with probability one that the true process generating 
observations on the latent component of productivity is the low persistence process. Notice, though that even if the agent were to know the value of of the persistence parameter $\rho$ (i.e., $\eta=1$ or 0 ), there is still ambiguity, since $x_{t}$ is still unknown. However, once $\rho$ is fixed, the ambiguity is fixed too, since the volatility of a degenerate mixture distribution is a given constant. In this case, both $\Lambda_{t}$ and $\xi_{t}$ are different from one and the parameter $\alpha$ represents the uncertainty aversion by the consumer ${ }^{17}$ If, in addition, $x_{t}$ is observed, then both $\Lambda_{t}$ and $\xi_{t}$ are one and the consumer's problem is an expected utility maximization problem.

- $\alpha \rightarrow 0$. This corresponds to the case of ambiguity neutral preferences where, from equation (3.4), the agent now behaves as a pure Bayesian decision-maker who is uncertain about the temporary component of TFP growth $x_{t}$, and has beliefs that are just a mixture of the probability distributions for $x_{i t}, i=l, h$. In this case, the distortion functions $\Lambda_{t}$ and $\xi_{t}$ just converge to one.

To further understand these results, note that $\Lambda_{t}$ does not affect the second-order distributions appearing in the optimality conditions 3.10 3.11). In these expressions, $\xi_{i, t}$ depends on expectations that are taken with respect to the distribution of $x_{i, t}$, conditional on information of the history the technology shock $\left(g_{A, t}, g_{A, t-1}, \cdots, g_{A, 0}\right)$ and, hence, is random from the view of the agent's subjective beliefs at date $t$. Thus, the functions $\xi_{i, t}$ may be viewed as the factor that creates the endogenous tilting or distortion in agents' beliefs due to ambiguity and ambiguity aversion. In the case of ambiguity aversion with $\alpha>\gamma>0$, the distortion puts greater weight (relative to a pure Bayesian decision-maker) on the probability distributions of the $x_{i, t}$ that are associated with lower expected continuation values, $E_{x_{i, t}} \hat{J}\left(\hat{k}_{t+1}^{(i)}, \hat{x}_{h, t+1}^{(i)}, \hat{x}_{l, t+1}^{(i)}, \eta_{t+1}^{(i)}\right)$ for $i=l, h$. Thus, we may view the impact of the $\xi_{i, t}$ as shaping the "as if" beliefs of the agent, that is, the (probabilistic) belief that supports the action chosen in equilibrium. The quantitative findings that follow discuss how such endogenous pessimism affects the cyclical dynamics of the endogenous variables in the model.

\footnotetext{
${ }^{17}$ For a similar setup, see Backus et al. $(2015)$.
} 
Table 2: Unconditional business cycle moments

Full Sample - 1947:1-2015:4

Restricted Sample - 1978:1-2015:4

\begin{tabular}{|c|c|c|c|c|c|c|c|c|c|c|}
\hline & \multicolumn{5}{|c|}{ Investment-Output Ratio, $i / y$ : 0.143} & \multicolumn{5}{|c|}{ Investment-Output Ratio, $i / y: 0.157$} \\
\hline & \multicolumn{10}{|c|}{ Standard deviations } \\
\hline & $y$ & $c$ & $i$ & $n$ & $p$ & $y$ & $c$ & $i$ & $n$ & $p$ \\
\hline & 1.624 & 1.262 & 7.442 & 1.925 & 0.934 & 1.294 & 1.076 & 6.284 & 1.783 & 0.930 \\
\hline & \multicolumn{10}{|c|}{ Correlations } \\
\hline & $y$ & $c$ & $i$ & $n$ & $p$ & $y$ & $c$ & $i$ & $n$ & $p$ \\
\hline$y$ & 1.000 & 0.774 & 0.841 & 0.886 & -0.064 & 1.000 & 0.866 & 0.913 & 0.865 & -0.266 \\
\hline$c$ & & 1.000 & 0.694 & 0.658 & -0.068 & & 1.000 & 0.730 & 0.768 & -0.267 \\
\hline$i$ & & & 1.000 & 0.762 & -0.100 & & & 1.000 & 0.817 & -0.296 \\
\hline$n$ & & & & 1.000 & -0.539 & & & & 1.000 & -0.715 \\
\hline$p$ & & & & & 1.000 & & & & & 1.000 \\
\hline
\end{tabular}

Note: This table shows the average investment-output ratio and the unconditional second moments of the HP-filtered series on output, $y$, consumption, $c$, investment, $i$, hours worked, $n$, and labor productivity, $p$.

\section{Quantitative findings}

In this section, we present simulations of the smooth ambiguity model under different parameter values and with varying degrees of prior ambiguity, or alternatively, "confidence". These provide a benchmark to compare against the results from simulating the model by Ilut and Schneider (2014) in Section 5 .

\subsection{Business cycle moments}

Table 2 presents the unconditional business cycle moments for the full sample between 1947:1-2015:4 and the restricted sample between 1978:1-2015:4. All series are Hodrick-Prescott filtered versions of the original series. The data on output, consumption, investment, and hours worked are obtained from the Federal Reserve Bank of St. Louis database (FRED). The output, $y$, consumption, $c$, and investment, $i$ series are seasonally adjusted and measured in chained 2009 dollars. Investment refers to total private investment and the hours worked series is an index of total hours worked in the nonfarm business sector, seasonally adjusted with the 2009 value equal to 100 .

Table 2 shows the standard business cycle facts regarding postwar US cyclical fluctuations. There are differences between the results for the full sample and the restricted sample, in line with the 
"Great Moderation" hypothesis that we alluded to earlier; see, for example, Stock and Watson (2002). In what follows, we seek to understand how well the Real Business Cycle model with ambiguity and ambiguity aversion can account for these facts.

\subsection{Simulation approach}

The unconditional moments for all of the series are computed by randomly drawing a sample of shocks from the high and low persistence processes to generate a pseudo observation on the growth rate of technology, $g_{A, t+1}$, at each date. However, the agent does not know which process the realization of the technological growth shock is coming from and must make inferences from observations on the growth rate of technology about the nature of the process from which such observations are drawn. Given initial conditions $\hat{k}_{0}=k_{s}, \hat{x}_{l, 0}=0, \hat{x}_{h, 0}=0, \eta_{0}=0.5$, the laws of motion for the capital together with the Kalman filter are used to determine the evolution of the capital stock and beliefs along the agent's optimal path. These constitute the endogenous state variables for the model. ${ }^{18}$ Given the simulated values of $\hat{i}_{t}, n_{t}$ and $g_{A, t+1}$, output and consumption today together with next period's capital stock are obtained from the production function, the resource constraint and the law of motion for capital. To generate next period's beliefs which will form next period's state vector, current beliefs $\left(\hat{x}_{l, t}, \hat{x}_{h, t}, \eta_{t}\right)$ are updated given the new observation $g_{A, t+1}$ using the Kalman filter.

The simulated solutions for the transformed problem are used to generate levels for the nonstationary series, which are then detrended using a Hodrick-Prescott filter for quarterly series. The simulated moments are based on 1,000 simulations of 400 periods, with a burn-in sample of 200 periods. In these simulations, the estimates of the technology shock processes are obtained by using uninformative priors as reported in Table 1. In our model solution, the decision rules for optimal investment and hours depend nonlinearly on the latent beliefs $\left(\hat{x}_{h t}, \hat{x}_{l t}, \eta_{t}\right)$. Hence, to prevent any specific shock sequence from affecting the simulations, the same set of shocks are used for all of the simulations reported in the paper.

\footnotetext{
${ }^{18}$ The decision rules used to simulate the response of investment and hours to different shock processes are obtained as grids of values as part of the solution for the indirect value function $\hat{J}$, which are then projected on the Chebyshev polynomials evaluated at the current state $\left(\hat{k}_{t}, \hat{x}_{l, t}, \hat{x}_{h, t}, \eta_{t}\right)$.
} 
Table 3: Parameter Values

\begin{tabular}{lll}
\hline$\beta$ & Subjective discount factor & 0.988 \\
$\gamma$ & Coefficient of risk aversion & $0.5,0.8$ \\
$\alpha$ & Coefficient of ambiguity aversion & $0.8,5$ \\
$a$ & Capital share & 0.3 \\
$\delta$ & Depreciation rate & 0.025 \\
\hline
\end{tabular}

Note: This table reports parameter choices used in the simulations of the models. The calibration is at the quarterly frequency. The share of leisure is implicitly computed from the intratemporal marginal rate of substitution between consumption and leisure, assuming a steady value of hours worked $n_{s s}=1 / 3$.

\subsection{Baseline results}

The parameter values used in the simulations are standard to the business cycle literature. Specifically, the capital share is set at 0.3 and the depreciation rate at the quarterly frequency at 0.025 . The share of leisure in preferences denoted by the parameter $\nu$ is given by 0.3663 . This is based on the steady state values of the model where the share of working time is set at $1 / 3$, consistent with the finding that households spend one-third of their time working. The modified discount factor is given by $\beta \exp (\nu(1-\gamma) \bar{g})$ and depends on the values of the preference and productivity parameters. The estimation results provided in Table 1 imply that the average growth rate of the technology shock at the quarterly frequency is $0.47 \%$, which is slightly higher but consistent with the average growth rate of technology based on the model reported in Jahan-Parvar and Liu (2014) as well as earlier estimates in Kaltenbrunner and Lochstoer (2010) and Croce (2014).

In the smooth model of ambiguity, ambiguity aversion is modeled by the concavity of the function $\phi(\cdot)$, and is measured by the parameter $\alpha$ while risk aversion, as usual, is inferred from the properties of the $u(\cdot)$, and is measured by the parameter $\gamma$. However, the specification of preferences employed here does not allow for a separation of risk aversion and the intertemporal elasticity of substitution (IES). In other recent analyses which allow for the separation of risk aversion and intertemporal substitution, the intertemporal elasticity of substitution (IES) is typically set to be greater than one ${ }^{19}$ An IES greater than one corresponds to a value of $\gamma$ less than one in our specification. In our initial quantitative analysis, we employ the values of $(\gamma=0.5, \alpha=0.8)$, which allows for an IES

\footnotetext{
${ }^{19}$ For evidence on this point, see Vissing-Jørgensen and Attanasio $(2003)$, though the evidence is mixed.
} 
greater than unity and satisfies the restriction that $\gamma<\alpha$.

Table 4 displays the results shows the simulation results for alternative parameter values. While the simulations are conducted separately for the low persistence/high variance and high persistence/low variance processes by drawing realizations of the shocks from these processes, the agent is assumed not to know which process the relazations are coming from. The results in Table 4 show that the standard business cycle facts hold in our model. Here (i) the volatility of investment is nearly three times that of output, (ii) output growth is more variable than consumption, and (iii) the magnitudes of the simulated standard deviations match the standard deviations of the actual series for the post-1980's sample period. However, the model does much more poorly in matching the variability of hours, which is a standard result in the business cycle literature; see Hansen (1985) or Altug (1989). It also understates the volatility of consumption observed in the data.

A second experiment might involve altering the value of the ambiguity aversion parameter, $\alpha$, holding constant the value of $\gamma$ at 0.5 . In the literature, a variety of values have been assumed for the ambiguity aversion parameter in models with smooth ambiguity; for example, Ju and Miao (2012) use thought experiments in a specification that allows for a three-way separation between risk aversion, intertemporal substitution and ambiguity aversion to set the ambiguity aversion parameter at 8.864 together with an IES equal to 1.5. In other studies, the ambiguity aversion parameter is chosen to match certain moments in the data. As examples, Collard et al. (2018) choose the ambiguity aversion parameter to match the risk-free rate of 1.5\%, averaged over the period 1978-2011 while Liu and Zhang (2018) match the mean equity premium in the data by setting the ambiguity aversion parameter at 45 together with an IES of 1.5 and a coefficient of relative risk aversion of 5 .

In a purely business cycle context, Tallarini (2000) and Backus et al. (2015) have shown that impact of uncertainty/ambiguity aversion on quantities in real business cycle models of the type studied here is minor compared to its impact on prices and in particular, on the risk-free interest rate ${ }^{20}$ To examine this hypothesis numerically, we simulated the model for alternative values of $\alpha$ ranging from 5 to 50 for a value of $\gamma=0.5$. For the baseline model, we found small effects on the simulated moments for values of $\alpha$ around 50. As a counterfactual exercise, we also simulated the

\footnotetext{
${ }^{20}$ This result is known to follow from the complete markets assumption that underlies the allocations of the social planning problem and the precautionary saving motives that it induces.
} 
Table 4: Simulation Results: Ambiguity about the Persistence/Volatility of the Unobserved Component of TFP Growth

\begin{tabular}{|c|c|c|c|c|c|c|c|c|c|c|}
\hline & \multicolumn{5}{|c|}{$\gamma=0.5, \alpha=0.8$} & \multicolumn{5}{|c|}{$\gamma=0.8, \alpha=5$} \\
\hline & \multicolumn{9}{|c|}{ Simulations conditional on $\rho_{k}=0.85$} & \\
\hline & \multicolumn{10}{|c|}{ Standard deviations } \\
\hline & $y$ & $c$ & $i$ & $n$ & $p$ & $y$ & $c$ & $i$ & $n$ & $p$ \\
\hline & 1.362 & 0.601 & 6.398 & 0.715 & 0.775 & 1.233 & 0.596 & 5.093 & 0.528 & 0.775 \\
\hline \multicolumn{11}{|c|}{ Correlations } \\
\hline & $y$ & $c$ & $i$ & $n$ & $p$ & $y$ & $c$ & $i$ & $n$ & $p$ \\
\hline$y$ & 1.000 & 0.663 & 0.939 & 0.906 & 0.921 & 1.000 & 0.858 & 0.952 & 0.920 & 0.964 \\
\hline$c$ & & 1.000 & 0.368 & 0.283 & 0.902 & & 1.000 & 0.662 & 0.588 & 0.964 \\
\hline$i$ & & & 1.000 & 0.994 & 0.733 & & & 1.000 & 0.994 & 0.837 \\
\hline$n$ & & & & 1.000 & 0.669 & & & & 1.000 & 0.783 \\
\hline$p$ & & & & & 1.000 & & & & & 1.000 \\
\hline$i / y$ & & & & & 0.1758 & & & & & 0.1715 \\
\hline \multicolumn{11}{|c|}{ Simulations conditional on $\rho_{k}=0.30$} \\
\hline & \multicolumn{10}{|c|}{ Standard deviations } \\
\hline & $y$ & $c$ & $i$ & $n$ & $p$ & $y$ & $c$ & $i$ & $n$ & $p$ \\
\hline & 1.343 & 0.592 & 6.315 & 0.706 & 0.764 & 1.222 & 0.589 & 5.058 & 0.524 & 0.767 \\
\hline & \multicolumn{10}{|c|}{ Correlations } \\
\hline & $y$ & $c$ & $i$ & $n$ & $p$ & $y$ & $c$ & $i$ & $n$ & $p$ \\
\hline$y$ & 1.000 & 0.661 & 0.939 & 0.907 & 0.921 & 1.000 & 0.858 & 0.953 & 0.921 & 0.964 \\
\hline$c$ & & 1.000 & 0.368 & 0.283 & 0.900 & & 1.000 & 0.664 & 0.590 & 0.964 \\
\hline$i$ & & & 1.000 & 0.994 & 0.735 & & & 1.000 & 0.994 & 0.839 \\
\hline$n$ & & & & 1.000 & 0.670 & & & & 1.000 & 0.784 \\
\hline$p$ & & & & & 1.000 & & & & & 1.000 \\
\hline$i / y$ & & & & & 0.1756 & & & & & 0.1676 \\
\hline
\end{tabular}

Note: The model is simulated based on the decision rules for the main model with ambiguity where the agent cannot distinguish perfectly between two processes with persistence $\rho_{l}=0.30$ and $\rho_{h}=0.85$. The parameters characterizing the shock processes are derived from the estimates in Table 1 and assume that $\bar{g}_{h}=0.00469$ and $\rho_{h}=0.85, \sigma_{A_{h}}=0.00952, \sigma_{x_{h}}=0.0004$ for the high persistence process and $\rho_{l}=0.30, \sigma_{A_{l}}=0.00946, \sigma_{x_{l}}=0.00044$ for the low persistence process. 
model using the TFP series for a specific industry that displays significant idiosyncratic uncertainty using sectoral data from Bloom et al. (2018). There the impact of ambiguity aversion is picked up for values of $\alpha$ as low as 5.21 Backus et al. (2015) also find that changing the ambiguity aversion parameter in a business cycle model with risk and ambiguity has minimal effects; see their Table $3 .{ }^{22}$ Despite its minor effects, increasing the value of $\alpha$ reduces the volatility and increases the persistence of the quantity series without changing the overall magnitudes significantly compared to the case with $\gamma=0.5, \alpha=0.8$. To enable agents to smooth their responses to shocks, the impact of greater ambiguity aversion is to increase the optimal level of investment and hours worked ${ }^{23}$

Hence, as a conservative estimate, we consider a value of $\alpha$ as 5 . The second panel of Table 4 increases the value of $\gamma$ to 0.8 after setting the value of $\alpha$ to 5 , which ensures that $\gamma<\alpha$. In this case, there is a substantial decline in the volatility of output, investment and hours worked, and an increase in the comovement of the different series. Since the ambiguity aversion parameter has minor effects on these moments, this finding may be attributed to an increase in risk aversion and a decline in intertemporal substitution. To the extent that an increase in $\gamma$ reflects the decline in the willingness of consumers to substitute consumption across periods, the volatility of all of the key macroeconomic aggregates declines and their co-movement increases.

It might be helpful to compare, at an intuitive level, the mechanisms underlying the move toward precautionary saving (alternatively, the increase in demand for the risk free asset) under risk aversion with that under ambiguity aversion. As argued, an increase in ambiguity aversion $(\alpha)$ works through a distortion of the effective probability distribution, which arises through the effect of the functions $\xi_{i t}, i=l, h$ described in Section 3.3. Such a distortion increases the weights on those future states of the world where incomes are lower. Since the agent is risk averse, marginal utility in such states are greater and so she will shift consumption to those states. Increasing (precautionary) demand for the risk free asset allows her to do this, leading to a lower risk free rate in equilibrium.

An increase in risk aversion $(\gamma)$ increases the marginal utility in states of the world where incomes

\footnotetext{
${ }^{21}$ Section C of the Supplementary Material describes the estimated TFP process for this industry, namely, Petroleum Mining, while Section D describes the nature of the decision rules and filtered beliefs.

${ }^{22}$ Similar findings about the minor impact of changes in the ambiguity aversion parameter are reported by Jahan-Parvar and Liu (2014) and Liu and Zhang (2018).

${ }^{25}$ See also Cagetti et al. $(2002)$, who show that a robust motive for precautionary saving increases the capital stock in a one-sector optimal growth model.
} 
are lower, pushing the agent to shift consumption to those states. However, unlike the case of increase in $\alpha$, there is a countervailing effect. Given that expected growth is positive, the agent is relatively poorer today compared to the expected income tomorrow. Thus an increase in $\gamma$ also prompts the agent to consume more today. This explains the absence of intertemporal consumption smoothing at higher values of $\gamma$, which also leads to the lower volatility and greater persistence of the quantity series for $\gamma=0.8$ as opposed to $\gamma=0.5$.

We further examine the behavior of risk free rate to quantitatively illustrate the effects of precautionary saving motive; see Section D of the Supplementary Material. As argued, ambiguity and ambiguity aversion affect the risk-free through the distortion functions $\xi_{i, t}$ for $i=l, h$ which, in turn, operate through two mechanisms. The first is through the endogenous doubt and pessimism induced by these distortion functions. The second is through learning about the persistence of the underlying TFP process, which occurs because such learning leads to time-varying uncertainty ${ }^{24}$

Finally, the behavior of the investment-output ratio provides another gauge of the impact of the different features of the model. This is close to the value reported for the data, and suggests that the model is able to reconcile the average value of 0.157 for the post-1980 period. Having explained the impact of the different parameters in generating the cyclical dynamics of key macroeconomic aggregates, the next section now turns to a discussion of the effects of changes in ambiguity directly on such moments.

\subsection{The role of changes in initial ambiguity or "confidence"}

How do changes in ambiguity or "confidence" affect the business cycle moments? This section asks how the conclusions would change if there is a lower degree of ambiguity compared to the baseline model. In the model proposed by Ilut and Schneider (2014), agents have belief sets that describe confidence about the evolution of future TFP, and they are assumed to gather intangible information about the ambiguous component of TFP. In their framework, shocks to confidence are modeled as

\footnotetext{
${ }^{24}$ For the aggregate TFP process, the results in Section D of the Supplementary Material show that such time-varying uncertainty is small, leading to lower distortions and hence, a smaller precautionary saving effect coupled with a relatively high risk-free rate. For an industry such as Petroleum Mining where idiosyncratic time-varying uncertainty is high, there are greater distortions under ambiguity and ambiguity aversion, leading to a lower risk-free rate in equilibrium.
} 
exogenous changes in ambiguity. In their empirical analysis, these authors bound agents' belief sets regarding the mean of aggregate TFP using data from the Survey of Professional Forecasters (SPF) on forecast dispersion. Other papers that use survey data to identify agents' beliefs include Bhandari et al. (2016), who consider the robust preference model of Hansen and Sargent (2001) and extend the model to allow agents to be exposed to shocks about their ambiguity concerns or Rossi et al. (2016), who discuss empirical approaches for constructing aggregate measures of ambiguity/Knightian uncertainty. Bhandari et al. (2016) use household forecasts of key macroeconomic variables from the University of Michigan Surveys of Consumers relative to the SPF forecasts as belief wedges while Rossi et al. (2016) use measures of the forecast density from the SPF. In this section, we provide a measure of confidence based on changes in the initial prior beliefs of agents.

The effect of ambiguity evolves endogenously in our model as a function of the distorted beliefs $\xi_{i, t}$ for $i=l, h$ introduced in Section 3.3. This is in contrast to other frameworks where changes in ambiguity are exogenously determined. Nevertheless, it is possible to specify a notion of changes in initial ambiguity through the initial prior beliefs of agents. In our model, agents' beliefs about the first-order distribution characterizing the transitory component of the productivity process depend on parameter values such as the persistence or the volatility of the unknown latent process governing TFP growth, $x_{t}$. As the agent is a Bayesian learner, more informative prior beliefs/distributions on such model parameters may potentially help to reduce the agent's (second-order) uncertainty about the specific data generating process. Hence, initial priors that increase the signal-to-noise ratio (computed by the ratio of the variances of transitory and permanent components, $\sigma_{x}^{2} / \sigma_{g}^{2}$ ) could improve the agent's inference on the transitory component. The increasing informativeness of the transitory process, in turn, could increase the ability of the agent to discriminate between the low and high persistent processes. We may thus view such changes in initial priors as a measure of changes in initial ambiguity by agents regarding the true data generating process.

Table 5 provides simulation results regarding the role of changes in initial ambiguity or varying degrees of confidence. To derive these results, we re-estimate the unknown TFP processes by the use of priors that increase the signal-to-noise ratio in the data. An increase in confidence by agents is 
reflected in the evolution of the beliefs $\eta_{t}$ approaching to values closer to unity 25 The new decision rules are calculated under the assumption that agents face unknown TFP processes estimated using progressively more informative priors displayed in Panels B and E of Table C.1 in the Supplementary Material. However, these decision rules are simulated under the original TFP process as the actual data generating process. The parameter values $\gamma=0.5, \alpha=0.8$ are used in all the simulations.

Table 5 shows that lower initial ambiguity, or put differently, a higher level of confidence, about the process governing the cyclical component of TFP reduces the volatility of optimal investment and hours choices. Considering the Panel B estimates in Table 5, the volatility of investment and hours worked decreases between 10\% (11\%) compared to the baseline results. Output and consumption volatility also decrease but by smaller amounts on the order of $4 \%(2 \%)$, respectively ${ }^{26}$ In all cases, greater informativeness about the underlying TFP process also increases the co-movement among the different series. Another way to understand these results is that they reflect the impact of learning under greater information. More informative initial priors in the estimation of the temporary component of the underlying TFP process lead to larger standard deviations for the shocks to the unknown cyclical component of the technological growth $x_{k, t}, \sigma_{k}$, for $k=l, h$, in Table 5 compared the standard deviations for the same shocks in Table 4. Thus, agents are better able to infer the unknown temporary component of technology growth and to act on this knowledge to smooth their optimal investment and hours choices ${ }^{27}$ These results imply that changes in confidence coupled with learning dynamics can have significant effects on the business cycle moments implied by the model, suggesting a link with other models that study similar effects.

\footnotetext{
${ }^{25}$ See Figure C.1 in the Supplementary Material. The details of the different prior settings and the resulting evolution of beliefs, $\eta_{t}$ are displayed in the Supplementary Material, Section C.

${ }^{26}$ The Panel E estimates imply larger effects, with the volatility of output decreasing by $15 \%$ and the volatility of investment and hours worked falling by around 37\% (43\%) compared to the baseline results in Table 4 . In this case, there is a slight increase in consumption volatility compared to the Panel B estimates, which arises from the lessening of the precautionary motive in saving under lower ambiguity.

${ }^{27}$ In these experiments, since we keep the nature of the underlying TFP process used in the simulations constant, the effects on the business cycle moments are typically arising from the changes in the behavior of agents due to lower ambiguity, not changes in the data generating process.
} 
Table 5: Simulation Results: Informative Priors about the Persistence/Volatility of the Unobserved Component of TFP

\begin{tabular}{|c|c|c|c|c|c|c|c|c|c|c|}
\hline & \multicolumn{5}{|c|}{ Panel B } & \multicolumn{5}{|c|}{ Panel E } \\
\hline & \multicolumn{9}{|c|}{ Simulations conditional on $\rho_{k}=0.85$} & \\
\hline & \multicolumn{10}{|c|}{ Standard deviations } \\
\hline & $y$ & $c$ & $i$ & $n$ & $p$ & $y$ & $c$ & $i$ & $n$ & $p$ \\
\hline & 1.310 & 0.590 & 5.781 & 0.635 & 0.774 & 1.154 & 0.609 & 4.001 & 0.405 & 0.779 \\
\hline \multicolumn{11}{|c|}{ Correlations } \\
\hline & $y$ & $c$ & $i$ & $n$ & $p$ & $y$ & $c$ & $i$ & $n$ & $p$ \\
\hline$y$ & 1.000 & 0.756 & 0.946 & 0.913 & 0.943 & 1.000 & 0.951 & 0.975 & 0.951 & 0.987 \\
\hline$c$ & & 1.000 & 0.507 & 0.431 & 0.931 & & 1.000 & 0.859 & 0.809 & 0.988 \\
\hline$i$ & & & 1.000 & 0.994 & 0.785 & & & 1.000 & 0.995 & 0.926 \\
\hline$n$ & & & & 1.000 & 0.725 & & & & 1.000 & 0.889 \\
\hline$p$ & & & & & 1.000 & & & & & 1.000 \\
\hline$i / y$ & & & & & 0.1756 & & & & & 0.1755 \\
\hline \multicolumn{11}{|c|}{ Simulations conditional on $\rho_{k}=0.30$} \\
\hline & \multicolumn{10}{|c|}{ Standard deviations } \\
\hline & $y$ & $c$ & $i$ & $n$ & $p$ & $y$ & $c$ & $i$ & $n$ & $p$ \\
\hline & 1.293 & 0.581 & 5.728 & 0.630 & 0.763 & 1.144 & 0.598 & 4.020 & 0.409 & 0.767 \\
\hline & \multicolumn{10}{|c|}{ Correlations } \\
\hline & $y$ & $c$ & $i$ & $n$ & $p$ & $y$ & $c$ & $i$ & $n$ & $p$ \\
\hline$y$ & 1.000 & 0.753 & 0.947 & 0.913 & 0.942 & 1.000 & 0.946 & 0.974 & 0.950 & 0.986 \\
\hline$c$ & & 1.000 & 0.502 & 0.420 & 0.930 & & 1.000 & 0.849 & 0.798 & 0.987 \\
\hline$i$ & & & 1.000 & 0.995 & 0.782 & & & 1.000 & 0.995 & 0.922 \\
\hline$n$ & & & & 1.000 & 0.723 & & & & 1.000 & 0.883 \\
\hline$p$ & & & & & 1.000 & & & & & 1.000 \\
\hline$i / y$ & & & & & 0.1755 & & & & & 0.1754 \\
\hline
\end{tabular}

Note: The model is simulated based on the decision rules for the main model with ambiguity where the agent cannot distinguish perfectly between two processes with persistence $\rho_{l}=0.30$ and $\rho_{h}=0.85$. In Panel B, the parameters of the technology shock process are set so that $\rho_{h}=0.85, \sigma_{A_{h}}=0.00936, \sigma_{x_{h}}=0.00139$ for the high persistence process and $\rho_{l}=0.30$, $\sigma_{A_{l}}=0.00905, \sigma_{x_{l}}=0.00237$ for the low persistence process. In Panel E, the parameters of the technology shock process are set so that $\bar{g}_{h}=0.00469$ and $\rho_{h}=0.85, \sigma_{A_{h}}=0.00915$, $\sigma_{x_{h}}=0.00283$ for the high persistence process and $\rho_{l}=0.30, \sigma_{A_{l}}=0.00819, \sigma_{x_{l}}=0.00467$ for the low persistence process. 


\section{A New Keynesian model with maxmin expected utility}

Our analysis so far shows the key role that the evolution of beliefs and changes in confidence play in the Real Business Cycle model with ambiguity aversion. In an influential article, Ilut and Schneider (2014) present a New Keynesian model with maxmin expected utility that incorporates shocks to confidence. Such shocks to confidence are viewed as changes in ambiguity. The New Keynesian business cycle model that the authors use has been widely studied in the literature; see Christiano and Eichenabum (1992) or Smets and Wouters (2007) 28

In this section, we compare the quantitative implications of our model with those from the model with worst case beliefs presented by Ilut and Schneider (2014). The interest in comparing the results from this model with ours is that the maxmin expected utility model is a limiting case of the smooth ambiguity model as ambiguity aversion goes to infinity. Since the Ilut and Schneider (2014) model incorporates a variety of real and nominal frictions, our analysis will also allow us to compare the role that such frictions play in propagating ambiguity shocks.

\subsection{Belief sets and uncertainty shocks}

In their framework, Ilut and Schneider (2014) assume that the TFP shock follows a persistent AR(1) process as $\log \left(Z_{t+1}\right)=\rho \log \left(Z_{t}\right)+\mu_{t}^{*}+u_{t+1}$. They further assume that each innovation to TFP, $z_{t+1}^{x}=\log \left(Z_{t+1}\right)-\rho \log \left(Z_{t}\right)$, consists of two components defined as $u_{t}$, which is an i.i.d. normal sequence of innovations with mean zero and variance $\sigma_{u}^{2}$ and $\mu_{t}^{*}$, which is a deterministic sequence ${ }^{29}$ The conditional mean $\mu_{t}^{*}$ represents uncertainty in this model: even given large amounts of data, it is impossible to disentangle the effects of $\mu_{t}^{*}$ and the shocks sequence $u_{t}$. Hence, agents respond to such uncertainty by treating the parameter $\mu^{*}$ as ambiguous. Furthermore, their perception of ambiguity changes over time with the arrival of new information.

\footnotetext{
${ }^{28}$ The model features nominal rigidities in that consumers set wages for their specialized labor and intermediate goods producers set prices for their goods. Capital accumulation is subject to adjustment costs, and households experience disutility from working. Finally, the monetary policy authority sets the nominal interest rate according to a Taylor rule.

${ }^{29}$ The long-run behavior of $\mu_{t}^{*}$ is restricted so that its empirical moments converge to those of an i.i.d. normal stochastic process that has mean zero and variance $\sigma_{z}^{2}-\sigma_{u}^{2}>0$ and that is independent of $u_{t}$.
} 
The belief set of agents is parameterized by an interval of means centered around zero

$$
\log \left(Z_{t+1}\right)=\rho \log \left(Z_{t}\right)+\mu_{t}+u_{t+1}, \quad \mu_{t} \in\left[-a_{t},-a_{t}+2\left|a_{t}\right|\right]
$$

To complete this specification of beliefs, the variable $a_{t}$ is assumed to be an exogenous persistent process, interpreted as the cumulative effect of news that affect confidence, and it follows an $\operatorname{AR}(1)$ process as

$$
a_{t+1}-\bar{a}=\rho_{a}\left(a_{t}-\bar{a}\right)+\sigma_{a} \epsilon_{t+1}^{a},
$$

where $\epsilon_{t+1}^{*}$ is a standard normal i.i.d. innovation.

In this framework, ambiguity is the agent's uncertainty about the probability distribution governing the productivity process, which is represented by (2.4) in our setup. Here, the agent knows the distribution takes the form in (5.1); but, like our agent, does not know the mean of the distribution. The agent's uncertainty about this mean is the ambiguity: corresponding to the framework of the maxmin expected utility model, the ambiguity is expressed by making the mean interval-valued rather than a precise point value (there is no second-order distribution over the mean as in the smooth model). By exogenously changing the size of the interval (of values of the mean), it is possible to trace out comparative static exercises of changes in ambiguity. Ambiguity aversion is expressed here by evaluating policies by the worst case distribution, i.e., the distribution corresponding to minimum value in the considered interval of values of the mean.

\section{$5.2 \quad$ Simulation results}

We now consider the impact of such beliefs on a key set of aggregate variables in a New Keynesian Model with nominal and real frictions. In their analysis, Ilut and Schneider (2014) examine the behavior of growth rates of hours, consumption, investment, inflation, the nominal interest rate and a measure of dispersion for the period 1985:I-2011:I. The paper infers the dynamics of confidence from data on forecast dispersion available from the Survey of Professional Forecasters and uses linearized decision rules around a "zero risk steady state" that corrects for the fact that, from the perspective 
of the agent's worst case beliefs at time $t-1$, the average innovations to TFP are not zero at time $t$. They estimate the process for the ambiguity shock $a_{t}$ by assuming that average ambiguity is less than the total uncertainty about the process $Z_{t+1}{ }^{30}$

The analysis below considers several different versions of the New Keynesian model in Ilut and Schneider (2014) with TFP and confidence shocks ${ }^{31}$ The first one literally takes the parameters as estimated at the mode. The second one re-scales, uniformly, the volatility of both the TFP and the ambiguity shock such that the model replicates the volatility of output while a third specification re-scales the volatility of TFP to match the data. For each model, we consider three different versions. The first one, labeled as Benchmark, corresponds to Ilut and Schneider's full model. The second version shuts down nominal rigidities and therefore allows for a more fair comparison to our model. The last version shuts down real rigidities (adjustment costs, imperfect competition distortion) and makes the model directly comparable to ours. To make their results comparable to ours, we generate Hodrick-Prescott filtered versions of their series using the solution for growth rates from their model.

The results in Table 6 suggest that their Benchmark model is relatively successful at explaining the volatility of consumption and hours. This finding is based on a comparison of their model-generated moments with those in the data for the reduced sample in Table 2 . However, the volatility of output and investment is too low relative to the data in their framework 32 By contrast, our baseline framework reported in Table 4 matches the observed volatility of output and investment regardless of the parametrization that is considered. One reason why our model does better in matching output and investment volatility may be that we consider intertemporal elasticities of substitution (IES) to be greater than one for deterministic consumption paths whereas Ilut and Schneider (2014) assume a $\log$ utility function, which implies an IES is equal to unity. However, our model does more poorly in matching the volatility of consumption and more importantly, hours worked.

Strikingly, we find that removing the different types of frictions in their model has non-negligible effects on their results. When the nominal rigidities are eliminated, the results from the Benchmark

\footnotetext{
${ }^{30}$ Specifically, $\bar{a}=n \sigma_{z}$ and $\sigma_{a}=\sigma_{n} \sigma_{z}$, where $\sigma_{z}$ is the standard deviation of the innovations to productivity. In their Bayesian estimation, they assume beta distributions for $n$ and $\rho_{a}$ and an inverse gamma for $\sigma_{n}^{2}$.

${ }^{31}$ The authors also formulate a version of their model with shocks to the disutility of labor and TFP but this model is not of interest to our analysis.

${ }^{32} \mathrm{As}$ an example, the ratio of output (investment) volatility from their model and the data is $0.923 / 1.294=0.713$ $(2.568 / 6.284=0.409)$.
} 
model deteriorate considerably. In particular, the volatility of consumption, output, and investment fall by 35\%, 47\% and 60\%, respectively, and the volatility of hours worked nearly collapses. In the New Keynesian model with nominal price rigidities, the precautionary savings effects due to an increase in ambiguity do not lead to a decline in prices, implying that consumption volatility remains higher than with flexible prices. The volatility in hours arises from the aggregate demand effects of ambiguity: when ambiguity increases, prices do not adjust to take into account the lower productivity. Hence, aggregate demand must fall to accommodate the higher prices, which leads to larger declines in hour worked and increases the volatility of hours. These effects explain the relatively high volatility of consumption and hours in their Benchmark specification.

More importantly, a version of their model without nominal and real frictions which corresponds more closely to our RBC framework has limited ability at matching the moments for the quantity variables with the exception of investment. This series becomes more variable due to the absence of adjustment costs which is present in the other two specifications 33 When the volatility of TFP and ambiguity shocks are re-scaled to replicate the volatility of output, the volatility of the different series are more in line with the volatility of the series for the full sample reported in Table 2 Nevertheless, removing the nominal or real rigidities makes the performance of the model worse. Finally, considering a version of their model in which the volatility of TFP is re-scaled to match the data, the Benchmark model performs best in terms of explaining the volatility of hours worked, attesting to the role of the New Keynesian model rigidities in generating cyclical variation in this series. When these are removed as in the middle panel of Table 6, the performance of the model deteriorates on almost all dimensions.

\footnotetext{
${ }^{33}$ While there are some differences in the degree of the intertemporal elasticity of substitution (IES) that we assume, the differences in the business cycle moments when we change the parameter $\gamma$ are not large enough to account for the differences between our results and those of Ilut and Schneider (2014) without any frictions.
} 
Table 6: Business Cycle Moments in the Ilut and Schneider Model

\begin{tabular}{|c|c|c|c|c|c|c|c|c|c|c|c|c|c|c|c|}
\hline & \multicolumn{15}{|c|}{ Original Model } \\
\hline & \multicolumn{5}{|c|}{ Benchmark } & \multicolumn{5}{|c|}{ No Nominal Rigidities } & \multicolumn{5}{|c|}{ Frictionless } \\
\hline & \multicolumn{15}{|c|}{ Standard Deviations } \\
\hline & $y$ & $c$ & $i$ & $n$ & $p$ & $y$ & $c$ & $i$ & $n$ & $p$ & $y$ & $c$ & $i$ & $n$ & $p$ \\
\hline & 0.923 & 0.862 & 2.568 & 0.886 & 0.576 & 0.487 & 0.558 & 1.051 & 0.108 & 0.550 & 0.654 & 0.219 & 2.892 & 0.252 & 0.422 \\
\hline & \multicolumn{15}{|c|}{ Correlations } \\
\hline$y$ & \multirow[t]{5}{*}{1.000} & 0.899 & 0.893 & 0.792 & 0.376 & \multirow[t]{5}{*}{1.000} & \multirow{5}{*}{$\begin{array}{l}0.938 \\
1.000\end{array}$} & 0.826 & -0.516 & 0.986 & \multirow[t]{5}{*}{1.000} & \multirow{5}{*}{$\begin{array}{l}0.677 \\
1.000\end{array}$} & \multirow{5}{*}{$\begin{array}{l}0.986 \\
0.544 \\
1.000\end{array}$} & \multirow{5}{*}{$\begin{array}{l}0.953 \\
0.424 \\
0.990 \\
1.000\end{array}$} & 0.984 \\
\hline$c$ & & 1.000 & 0.607 & 0.753 & 0.277 & & & 0.579 & -0.781 & 0.982 & & & & & 0.799 \\
\hline$i$ & & & 1.000 & 0.666 & 0.397 & & & 1.000 & 0.054 & 0.721 & & & & & 0.939 \\
\hline$n$ & & & & 1.000 & -0.261 & & & & 1.000 & -0.651 & & & & & 0.883 \\
\hline$p$ & & & & & 1.000 & & & & & 1.000 & & & & & 1.000 \\
\hline & \multicolumn{15}{|c|}{ Re-scaled Model to match st. deviation of $y$} \\
\hline & \multicolumn{15}{|c|}{ Standard Deviations } \\
\hline & $y$ & $c$ & $i$ & $n$ & $p$ & $y$ & $c$ & $i$ & $n$ & $p$ & $y$ & $c$ & $i$ & $n$ & $p$ \\
\hline & 1.624 & 1.512 & 4.439 & 1.628 & 0.805 & 0.669 & 0.766 & 1.446 & 0.148 & 0.755 & 0.902 & 0.328 & 4.049 & 0.359 & 0.579 \\
\hline & \multicolumn{15}{|c|}{ Correlations } \\
\hline$y$ & \multirow[t]{5}{*}{1.000} & 0.910 & 0.901 & 0.874 & 0.244 & \multirow[t]{5}{*}{1.000} & \multirow{5}{*}{$\begin{array}{l}0.937 \\
1.000\end{array}$} & 0.825 & -0.513 & 0.986 & \multirow[t]{5}{*}{1.000} & \multirow{5}{*}{$\begin{array}{l}0.582 \\
1.000\end{array}$} & \multirow{5}{*}{$\begin{array}{l}0.980 \\
0.408 \\
1.000\end{array}$} & \multirow{5}{*}{$\begin{array}{l}0.936 \\
0.260 \\
0.988 \\
1.000\end{array}$} & \multirow{5}{*}{$\begin{array}{l}0.976 \\
0.745 \\
0.913 \\
0.837 \\
1.000 \\
\end{array}$} \\
\hline$c$ & & 1.000 & 0.641 & 0.827 & 0.159 & & & 0.576 & -0.780 & 0.982 & & & & & \\
\hline$i$ & & & 1.000 & 0.754 & 0.285 & & & 1.000 & 0.059 & 0.719 & & & & & \\
\hline$n$ & & & & 1.000 & -0.252 & & & & 1.000 & -0.649 & & & & & \\
\hline$p$ & & & & & 1.000 & & & & & 1.000 & & & & & \\
\hline & \multicolumn{15}{|c|}{ Re-scaled Model to match st. deviation of TFP } \\
\hline & \multicolumn{15}{|c|}{ Standard Deviations } \\
\hline & $y$ & $c$ & $i$ & $n$ & $p$ & $y$ & $c$ & $i$ & $n$ & $p$ & $y$ & $c$ & $i$ & $n$ & $p$ \\
\hline & 0.988 & 0.923 & 2.742 & 0.954 & 0.600 & 0.507 & 0.580 & 1.093 & 0.112 & 0.572 & 0.681 & 0.229 & 3.014 & 0.263 & 0.439 \\
\hline & & & & & & & & prrelatic & & & & & & & \\
\hline$y$ & 1.000 & 0.901 & 0.894 & 0.804 & 0.359 & 1.000 & 0.937 & 0.826 & -0.516 & 0.986 & 1.000 & 0.667 & 0.985 & 0.951 & 0.983 \\
\hline$c$ & & 1.000 & 0.612 & 0.764 & 0.263 & & 1.000 & 0.579 & -0.781 & 0.982 & & 1.000 & 0.530 & 0.407 & 0.793 \\
\hline$i$ & & & 1.000 & 0.679 & 0.383 & & & 1.000 & 0.054 & 0.721 & & & 1.000 & 0.990 & 0.936 \\
\hline$n$ & & & & 1.000 & -0.259 & & & & 1.000 & -0.651 & & & & 1.000 & 0.878 \\
\hline$p$ & & & & & 1.000 & & & & & 1.000 & & & & & 1.000 \\
\hline
\end{tabular}

Note: All model time series are HP-filtered. The original model uses the mode parameters of the Ilut-Schneider's model as reported in the paper. The first re-scaled model re-scales uniformly the volatility of the TFP and the ambiguity shock such that the Benchmark model reproduces the volatility of output in the data. The second re-scaled model re-scales uniformly the volatility of the TFP and the ambiguity shock such that the Benchmark model reproduces the volatility of TFP in the data. 
These results may be understood by noting that in the maxmin expected utility framework, agents make decisions based on the worst case distribution characterizing the TFP process. By contrast, agents in our framework have endogenously distorted beliefs represented by the factor $\xi_{i, t}$ for $i=l, h$ described in Section 3.3 that depend on the properties of the unknown distributions characterizing TFP growth. Evidently, the worst case scenario instills too much caution on the part of agents, who end up excessively reducing their response to shocks from the estimated TFP process. Since maxmin expected utility evaluation is obtained as a limiting case of the smooth ambiguity model when the ambiguity aversion parameter goes to infinity, this comparison may be thought of as an experiment that arbitrarily increases ambiguity aversion by agents ${ }^{34}$ Hence, in the absence of rigidities deriving from monopolistic competition in goods and labor supply and for price and wage setting by firms of intermediate goods and by households, respectively, the worst case beliefs that underlie their model specification lead to very low implied volatility in all of the quantity variables (with the possible exception of investment). By contrast, the endogenous distortions induced by our smooth model of ambiguity prove more capable of capturing most business cycle moments except for hours worked. This last result is due to the lack of any mechanism in our standard RBC model to capture the volatility in hours such as indivisible labor or search frictions.

\section{Other related models}

Other related models have also considered the role of ambiguity and ambiguity aversion in generating the cyclical variation of observed series.Bidder and Smith (2012) examine a Real Business Cycle model with multiplier preferences that reflect the agent's desire for robust policies following Hansen and Sargent (2008) and time-varying stochastic volatility shocks. In a robust decision-making problem framework where the agent seeks to minimize the distributions that twist continuation values towards outcomes that are painful to the agent, the minimization in a recursive representation of the problem yields a version of the log-exponential representation of the smooth ambiguity model that we discussed earlier. In their analysis, Bidder and Smith (2012) simulate the decision rules from

\footnotetext{
${ }^{34}$ Following Ju and Miao (2012), this result can be shown for log-exponential preferences studied in Section A of the Supplementary Material when $\alpha \rightarrow \infty$, which is from the class of preferences that displays constant absolute ambiguity aversion.
} 
the robust problem using the worst case distributions that account for the distortions arising from the agent's aversion to mis-specification. In our analysis, ambiguity is modeled in terms of the nature of the possible distributions for TFP growth that agents may face. Its effects are then determined from the optimal solution to an ambiguity averse agent's decision problem. Bidder and Smith (2012) find, among other results, that the model incorporating only shocks to time-varying volatility which is the source of endogenous pessimism in their framework is able to explain 1\%-16\% of the variability of such series as output, consumption, investment and hours compared to the full stochastic model that contains both volatility and technology shocks.

Bhandari et al. (2016) make use of the multiplier preferences that lead agents to make decisions on the basis of endogenously distorted pessimistic beliefs together with survey-based measures of ambiguity to account for labor market phenomena in a New Keynesian model with frictional labor markets. Their goal is to quantify the impact of ambiguity shocks on the joint dynamics of output, unemployment, inflation and interest rates as well as the households' belief wedges associated with these variables 35 They argue that their model can explain substantial variation in labor market variables such as the hiring rate as well as meaningful amount of common variation (up to $22 \%$ ) in the survey answers. Thus, their approach assumes that the agent and the econometrician use different sources of information in that households' survey responses are assumed to reflect their worst case beliefs. By contrast, our approach uses data on observed TFP and provides an analysis in which the agent and the econometrician have the same information in generating estimates of the true TFP process.

Leduc and Zheng (2016) consider a New Keynesian model with search and matching frictions in which the technology shocks display time-varying volatility. The distinguishing feature of their model is an option-value of a job match that arises from the irreversible nature of the employment that it represents. Higher uncertainty increases this option value and leads to an increase in unemployment as firms post fewer vacancies. By contrast, in the Real Business Cycle framework that we consider, an increase in uncertainty/ambiguity leads to an increase in precautionary saving, which reduces

\footnotetext{
${ }^{35}$ In their analysis, the theoretical versions of belief wedges are defined as the difference between survey responses from the Michigan Surveys of Consumers and model-based rational forecasts of future inflation and the unemployment rate. The authors measure belief wedges under the assumption that households provide answers regarding economic forecasts using the endogenous worst case probability distribution.
} 
both consumption and the real interest rate. In a model without search frictions, the option value effect on employment is absent, explaining why the standard RBC model cannot account adequately for the behavior of aggregate hours worked.

\section{Conclusion}

In this paper, we adopt the smooth ambiguity preferences of Klibanoff et al. $(2005,2009)$ to study the cyclical dynamics of a Real Business Cycle model with investment irreversibility and labor augmenting technology shocks. In comparison to recent papers that study such related questions, we seek to clarify the effect of changes in ambiguity and/or ambiguity aversion on dynamics of real variables in a neoclassical environment that is very minimally, if at all, perturbed by frictions, to better understand the effect of ambiguity in and of itself.

The recent literature on the impact of uncertainty and ambiguity has considered alternative forms of preferences to capture agents' aversion to bad outcomes in terms of worst case beliefs or preferences that lead to endogenously twisted beliefs weighing negative future outcomes more heavily. Such preferences have been embedded in standard Real Business Cycle frameworks or New Keynesian models with nominal and real rigidities and labor market frictions. Our analysis shows that the smooth ambiguity preferences proposed by Klibanoff et al. $(2005,2009)$ together with the endogenous sources of pessimism that this framework generates are able to account for the cyclical dynamics of key quantity variables that a model with worst case beliefs cannot capture. In earlier work, Collard et al. (2018) have shown how this framework may be used to rationalize the behavior of the historical equity premium. In this paper, we have further elucidated the transmission mechanisms inherent in this model and how they relate to other models of cyclical fluctuations under uncertainty/ambiguity. While our model lacks features such as labor market frictions that can account for labor market dynamics, our analysis shows that the use of smooth ambiguity preferences in a Real Business Cycle Model provides a flexible framework for accounting for many key stylized facts.

There are several ways in which this research could be extended in the future. First, the literature on accounting for business cycle phenomena with uncertainty/ambiguity has not explicitly tackled 
how various forms of market incompleteness may work towards generating a greater role for ambiguity and ambiguity aversion. As we argued above, the complete markets assumption is key to some of the results that are derived from this literature, including the strong precautionary savings effects of an increase in uncertainty/ambiguity. Second, an environment that considers individual heterogeneity may be more likely to capture the impact of uncertainty/ambiguity aversion and allow considerations of such issues as ambiguity trading. A third issue has to do with the measurement of TFP shocks in order to gauge their influence on business cycles. As we have shown above, time-varying uncertainty in TFP shocks is a key feature for uncovering the effects of ambiguity and ambiguity aversion. Hence, using more disaggregated measures of productivity at the firm or industry level which show more idiosyncratic variation (as in Bloom et al. (2018)) may provide a more useful approach to understanding the diffusion of endogenous pessimism in a general equilibrium economy with multiple sectors. We leave exploring these avenues for future work. 


\section{References}

Altug, S. (1989), Time-to-build and Aggregate Fluctuations: Some New Evidence, International Economic Review, 30, 889-920.

Altug, S. and P. Labadie (2008), Asset Pricing for Dynamic Economies, Cambridge University Press.

Backus, D., A. Ferriere, and S. Zin (2015), Risk and ambiguity in models of business cycles, Journal of Monetary Economics, 69, 42-63.

Bansal, R. and A. Yaron (2004), Risks for the Long-run: A Potential Resolution of Asset Pricing Puzzles, Journal fo Finance, 59, 1481-1509.

Bhandari, A., J. Borovicka, and P. Ho (2016), Identifying ambiguity shocks in business cycle models using survey data, Working paper, New York University.

Bianchi, F., C. L. Ilut, and M. Schneider (2018), Uncertainty Shocks, Asset Supply and Pricing over the Business Cycle, The Review of Economic Studies, 85, 810-854.

Bidder, R. and M. Smith (2012), Robust animal spirits, Journal of Monetary Economics, 59, 738-750.

Bloom, N., M. Floetotto, N. Jaimovich, I. Saporta-Eksten, and S. J. Terry (2018), Really Uncertain Business Cycles, Econometrica, 86, 1031-1065.

Boyarchenko, N. (2012), Ambiguity shifts and the 2007-2008 financial crisis, Journal of Monetary Economics, 59, 493-507.

Cagetti, M., L. Hansen, T. Sargent, and S. Williamson (2002), Robustness and Pricing with Uncertain Growth, Review of Financial Studies, 15, 363-404.

Christiano, L. and M. Eichenabum (1992), Current Real Business Cycle Theories and Aggregate Labor Market Fluctuations, American Economic Review, 82, 430-450.

Collard, F., S. Mukerji, K. Sheppard, and J.-M. Tallon (2018), Ambiguity and the historical equity premium, Quantitative Economics, 9, 945-993.

Collin-Dufresne, P., M. Johannes, and L. A. Lochstoer (2016), Parameter Learning in General Equilibrium: The Asset Pricing Implications, American Economic Review, 106, 664-98.

Croce, M. M. (2014), Long-run productivity risk: A new hope for production-based asset pricing?, Journal of Monetary Economics, 66, 13 - 31.

Cubitt, R., G. V. D. Kuilen, and S. Mukerji (2020), Discriminating between models of ambiguity attitude: A qualitative test, Journal of the European Economic Association, 18, 708-749.

Demers, F., M. Demers, and S. Altug (2003), Investment Dynamics, in S. Altug, J. Chadha, and C. Nolan (eds.), Dynamic Macroeconomic Analysis: Theory and Policy in General Equilibrium, Cambridge University Press, pp. 34-154.

Dimmock, S. G., R. Kouwenberg, O. S. Mitchell, and K. Peijnenburg (2016), Ambiguity Aversion and Household Portfolio Choice Puzzles: Empirical Evidence, Journal of Financial Economics, $119,559-577$. 
Epstein, L. and S. Zin (1989), Substitution, Risk Aversion, and the Temporal Behavior of Consumption and Asset Returns: A Theoretical Framework, Econometrica, 57, 937-69.

Fernald, J. G. (2012), A Quarterly, Utilization-adjusted Series on Total Factor Productivity, Working Paper Series 2012-19, Federal Reserve Bank of San Francisco.

Fisher, J. (2006), The Dynamic Effects of Neutral and Investment-Specific Technology Shocks, Journal of Political Economy, 114, 413-451.

Gilboa, I. and D. Schmeidler (1989), Maxmin Expected Utility with Non-unique Prior, Journal of Mathematical Economics, 18, 141-153.

Gordon, R. (2015), Secular Stagnation: A Supply-Side View, American Economic Review: Papers and Proceedings, 105, 54-59.

Greenwood, J., Z. Hercowitz, and P. Krusell (1997), Long-Run Implications of Investment-Specific Technological Change, American Economic Review, 87, 342-362.

Hansen, G. (1985), Indivisible Labor and the Business Cycle, Journal of Monetary Economics, 16, 309-327.

Hansen, L. (2007), Beliefs, Doubts, and Learning: Valuing Macroeconomic Risk, American Economic Review, 97, 1-30.

Hansen, L. and T. Sargent (2001), Robust Control and Model Uncertainty, American Economic Review, 91, 60-66.

Hansen, L. and T. Sargent (2008), Robustness, Pinceton University Press.

Ilut, C. and M. Schneider (2014), Ambiguous Business Cycles, American Economic Review, 104, 2368-99.

Jahan-Parvar, M. R. and H. Liu (2014), Ambiguity aversion and asset prices in production economies, The Review of Financial Studies, 27, 3060-3097.

Ju, N. and J. Miao (2007), Ambiguity, Learning, and Asset Returns, Working paper, Boston University.

Ju, N. and J. Miao (2012), Ambiguity, Learning, and Asset Returns, Econometrica, 80, 559-591.

Kaltenbrunner, G. and L. Lochstoer (2010), Long-run Risk through Consumption Smoothing, Review of Financial Studies, 23, 3190-3224.

Klibanoff, P., M. Marinacci, and S. Mukerji (2005), A Smooth Model of Decision Making under Ambiguity, Econometrica, 73, 1849-1892.

Klibanoff, P., M. Marinacci, and S. Mukerji (2009), Recursive Smooth Ambiguity Preferences, Journal of Economic Theory, 144, 930-976.

Kreps, D. and E. L. Porteus (1978), Temporal Resolution of Uncertainty and Dynamic Choice Theory, Econometrica, 46, 185-200. 
Leduc, S. and L. Zheng (2016), Uncertainty Shocks Are Aggregate Demand Shocks, Journal of Monetary Economics, 82, 20-35.

Liu, H. and Y. Zhang (2018), Financial Uncertainty with Ambiguity and Learning, Working paper, University of Manchester.

Nimark, K. (2014), Man-Bites-Dog Business Cycles, American Economic Review, 104, 2320-2367.

Rossi, B., T. Sekhposyany, and M. Soupre (2016), Understanding the Sources of Macroeconomic Uncertainty, Economic Working Paper Series 1531, University of Pompeu Fabra.

Smets, F. and R. Wouters (2007), Shocks and Frictions in US Business Cycles: A Bayesian DSGE Approach, American Economic Review, 97, 586-606.

Stock, J. and M. Watson (2002), Has the Business Cycle Changed and Why?, in M. Gertler and K. Rogoff (eds.), NBER Macroeconomics Annual, National Bureau of Economic Research.

Strzalecki, T. (2013), Temporal Resolution of Uncertainty and Recursive Models of Ambiguity Aversion, Econometrica, 81, 1039-1074.

Tallarini, T. (2000), Risk-sensitive Real Business Cycles, Journal of Monetary Economics, 45, 507-532.

Vissing-Jørgensen, A. and O. P. Attanasio (2003), Stock-Market Participation, Intertemporal Substitution, and Risk-Aversion, American Economic Review, 93, 383-391.

Zhou, J. (2015), The Good, the Bad, and the Ambiguous: The Aggregate Stock Market Dynamics around Macroeconomic News, Working paper, Harvard University. 